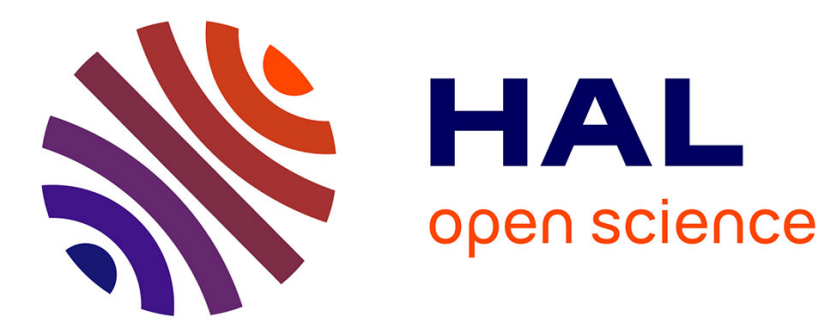

\title{
Study of a system for the isothermal separation of components in a binary alloy with change of phase
}

Daniel Kessler, Jean-Francois Scheid, Giulio Schimperna, Ulisse Stefanelli

\section{To cite this version:}

Daniel Kessler, Jean-Francois Scheid, Giulio Schimperna, Ulisse Stefanelli. Study of a system for the isothermal separation of components in a binary alloy with change of phase. IMA Journal of Applied Mathematics, 2004, 69 (3), pp.233-257. 10.1093/imamat/69.3.233 . hal-00141794

\author{
HAL Id: hal-00141794 \\ https://hal.science/hal-00141794
}

Submitted on 22 Jan 2021

HAL is a multi-disciplinary open access archive for the deposit and dissemination of scientific research documents, whether they are published or not. The documents may come from teaching and research institutions in France or abroad, or from public or private research centers.
L'archive ouverte pluridisciplinaire HAL, est destinée au dépôt et à la diffusion de documents scientifiques de niveau recherche, publiés ou non, émanant des établissements d'enseignement et de recherche français ou étrangers, des laboratoires publics ou privés. 


\title{
Study of a system for the isothermal separation of components in a binary alloy with change of phase
}

\author{
DANIEL KESSLER $†$ \\ Department of Mathematics, University of Maryland, College Park, MD 20742, USA \\ JEAN-FRANÇOIS SCHEID $\ddagger$ \\ Institut de Mathématiques Élie Cartan, Université de Nancy 1, F-54506 \\ Vandoeuvre-lès-Nancy Cedex, France \\ GIULIO SCHIMPERNA \\ Dipartimento di Matematica, Università di Pavia, Via Ferrata 1, I-27100 Pavia, Italy \\ AND \\ Ulisse STEFANELLIII \\ Istituto di Matematica Applicata e Tecnologie Informatiche del CNR, Via Ferrata 1, \\ I-27100 Pavia, Italy
}

[Received on 12 April 2002; revised on 23 December 2002]

\begin{abstract}
An isothermal model describing the separation of the components of a binary metallic alloy is considered. A process of phase transition is also assumed to occur in the solder; hence, the state of the material is described by two order parameters, i.e. the concentration $c$ of the first component and the phase field $\varphi$. A physical derivation is provided starting from energy balance considerations. The resulting system of PDEs consists of a rather regular second-order parabolic equation for $\varphi$ coupled with a fourth-order relation of Cahn-Hilliard type for $c$ with constraint and solution-dependent mobility. Global existence of solutions is proved and several regularity properties are discussed under more restrictive assumptions on the physical parameters. Continuous dependence on data is shown in a special case. An asymptotic analysis of the model is also performed, yielding at the limit step a coupling of the original phase field equation with a Stefan-like system for $c$.
\end{abstract}

Keywords: binary alloy; phase transition; fourth-order parabolic system; constraint; variational formulation; maximum principle; Faedo-Galerkin scheme.

\section{Introduction}

In this paper, we aim to study a model describing the diffusive separation of components in a binary metallic alloy possibly undergoing a phase transition phenomenon. As a basic simplification, the whole process is assumed to be isothermal. The system is then

\footnotetext{
†Email: kessler@math.umd.edu

‡Email: scheid@iecn.u-nancy.fr

§Email: giulio@dimat.unipv.it

IEmail: ulisse@ian.pv.cnr.it
} 
described on the one hand by the relative concentration $c$ of one component with respect to the mixture and on the other hand by a phase field parameter $\varphi$ which stands for the solidification state of the system $(\varphi=0$ indicates a solid phase whereas $\varphi=1$ is for a liquid phase). The functions $c$ and $\varphi$ satisfy the system

$$
\begin{aligned}
& \partial_{t} \varphi-\Delta \varphi=F_{1}(\varphi)+c F_{2}(\varphi), \\
& \partial_{t} c-\operatorname{div}(\mu(\varphi, c) \nabla w)=0, \\
& w \in-\Delta c+\beta(c)+\gamma(c)+g(\varphi),
\end{aligned}
$$

that will be coupled with the no-flux conditions and with the Cauchy conditions for $\varphi$ and $c$. Here, we have set $\beta:=\partial I_{[0,1]}$ the subdifferential of the indicator function of $[0,1]$; anyway, we point out that a wider class of constraints will be allowed in our analysis. The functions $F_{1}$ and $F_{2}$ are smooth and vanish at $\varphi=0$ and $\varphi=1$. This system is composed of a Cahn-Hilliard equation for the concentration (Cahn \& Hilliard, 1958) coupled with a second-order parabolic equation for the phase field function (Warren \& Boettinger, 1995; Wheeler et al., 1992). The mobility coefficient $\mu$ is allowed to depend on both $c$ and $\varphi$, but assumed to have a nondegenerate character as in Barrett \& Blowey (1999). In the rest of this section, we give the modelling leading to (1.1)-(1.3).

Modelling. Let us consider a binary mixture composed by two pure elements $A$ and $B$ which can be in both liquid and solid states inside a domain $\Omega$. The composition of the system is characterized by the relative concentration $c \in[0,1]$ of the component $B$ with respect to the mixture. The solidification state of the alloy is described by a phase field parameter $\varphi$ which is equal to 1 in a liquid phase and 0 in a solid phase. When $\varphi$ is strictly between 0 and 1, this indicates the presence of a mushy region. We do not take into account thermal effects, so the temperature $\theta$ of the system is assumed to be constant and fixed between the two melting temperatures $\theta_{m}^{A}$ and $\theta_{m}^{B}$ of the components $A$ and $B$. In that way, we consider that the system is fully determined by the knowledge of the scalar fields $c=$ $c(x, t)$ and $\varphi=\varphi(x, t)$ for each point $x \in \Omega$ at time $t$. Then, in order to obtain evolution equations for $c$ and $\varphi$, we introduce a Ginzburg-Landau type free energy depending on both the gradients of $c$ and $\varphi$ and also on a free energy density $f(\theta, c, \varphi)$. This total free energy $\mathcal{F}$ is given by Cahn \& Hilliard (1958)

$$
\mathcal{F}(\theta, c, \varphi)=\int_{\Omega}\left(\frac{\varepsilon_{\varphi}^{2}}{2}|\nabla \varphi|^{2}+\frac{\varepsilon_{c}^{2}}{2}|\nabla c|^{2}+f(\theta, c, \varphi)\right) \mathrm{d} x,
$$

where $\varepsilon_{\varphi}$ and $\varepsilon_{c}$ are given positive parameters. We assume the total mass of the system is conserved. Thus, denoting by $\mathbf{q}$ the mass flux, the mass balance equation reads

$$
\partial_{t} c+\operatorname{div} \mathbf{q}=0 \quad \text { in } \Omega,
$$

with the no-flux boundary condition

$$
\mathbf{q} \cdot \mathbf{n}=0 \quad \text { on } \partial \Omega,
$$

where $\mathbf{n}$ is the unit normal vector to the boundary $\partial \Omega$. Since we assume no external exchange, we also impose the boundary conditions

$$
\partial_{\mathbf{n}} \varphi=\partial_{\mathbf{n}} c=0 \quad \text { on } \partial \Omega \text {. }
$$


Now, we compute the time derivative of the total free energy. By the use of Green's formula with boundary conditions (1.6), (1.7) and recalling that the temperature $\theta$ is constant, we obtain

$$
\frac{\mathrm{d} \mathcal{F}}{\mathrm{d} t}=\int_{\Omega}\left(-\varepsilon_{\varphi}^{2} \Delta \varphi+\frac{\partial f}{\partial \varphi}\right) \partial_{t} \varphi+\nabla\left(-\varepsilon_{c}^{2} \Delta c+\frac{\partial f}{\partial c}\right) \cdot \mathbf{q} .
$$

Then, we have to introduce suitable constitutive laws for $\partial_{t} \varphi$ and $\mathbf{q}$. Namely, for suitable $M, \mu>0$, we assume

$$
\partial_{t} \varphi=-M \delta_{\varphi} \mathcal{F}=-M\left(-\varepsilon_{\varphi}^{2} \Delta \varphi+\frac{\partial f}{\partial \varphi}\right) \quad \text { in } \Omega
$$

and

$$
\mathbf{q}=-\mu \nabla \delta_{c} \mathcal{F}=-\mu \nabla\left(-\varepsilon_{c}^{2} \Delta c+\frac{\partial f}{\partial c}\right) \quad \text { in } \Omega,
$$

where, of course, $\delta_{\varphi} \mathcal{F}, \delta_{c} \mathcal{F}$ denote the first variations of $\mathcal{F}$ w.r.t. $\varphi, c$, respectively. Then, it is easy to see that the second principle of thermodynamics is satisfied; actually (1.8) entails $\mathrm{d} \mathcal{F} / \mathrm{d} t \leqslant 0$.

More in detail, we take $M$ as a positive constant (Kessler, 2001; Warren \& Boettinger, 1995), whereas $\mu=\mu(\varphi, c)$ is a positive function of $c$ and $\varphi$ which expresses a concentration and phase field dependent mobility. Indeed, the concentration dependence appears in the original derivation of the Cahn-Hilliard equation (Cahn \& Hilliard, 1958) and we shall discuss relevant choices for $\mu$ later on this section. Then, from the mass balance equation (1.5) together with (1.10) we deduce the equation for $c$

$$
\partial_{t} c+\operatorname{div}\left(\mu(\varphi, c) \nabla\left(\varepsilon_{c}^{2} \Delta c-\frac{\partial f}{\partial c}\right)\right)=0 \quad \text { on } \Omega .
$$

Now, let us turn to the free energy density $f$. This is supposed to be the sum of two contributions, namely

$$
f(\theta, c, \varphi)=\left[(1-c) f^{A}(\theta, \varphi)+c f^{B}(\theta, \varphi)\right]+j(c) .
$$

The first one arises as a convex combination of the free energies $f^{A}=f^{A}(\theta, \varphi)$ and $f^{B}=f^{B}(\theta, \varphi)$ of the pure components weighted by the concentration $c$; the second term is the potential energy $j$ of the mixing process (Kessler, 2001; Warren \& Boettinger, 1995), assumed to depend only on $c$. In contrast to Kessler (2001) and Warren \& Boettinger (1995), here $j$ might be nonconvex in order to describe a separation process of the two components. To be precise, our basic choice for $j$ is

$$
j(c)=I_{[0,1]}(c)+j_{0}(c),
$$

where $I_{[0,1]}$ is the indicator function of $[0,1]\left(I_{[0,1]}(c)=0\right.$ if $c \in[0,1], I_{[0,1]}(c)=+\infty$ otherwise) and $j_{0}$ is a regular function that possesses two local minima at $c=0$ and $c=1$. A typical form of $j_{0}$ is given for example by the double-well potential

$$
j_{0}(c)=16 \tau c^{2}(1-c)^{2},
$$


where $\tau>0$ is a given parameter (Elliott \& Garcke, 1996); anyway, we remark that other choices are possible (see e.g. Blowey \& Elliott, 1991, 1992; Elliott \& Garcke, 1996). The above setting, indeed, turns out to prescribe lower energy levels in presence of the pure states $A$ and $B$ than in the mixture. We point out that considering potentials with infinite barriers is a common choice when dealing with Cahn-Hilliard-like systems. Indeed, an expression like (1.13) is usually noted as a double obstacle potential. A potential of this kind was first proposed by Blowey \& Elliott $(1993,1994)$ and has been considered in several papers (e.g. Kenmochi et al., 1995, and references therein).

We remark that another and probably more usual expression for the potential is the logarithmic

$$
\chi(c)=c \log c+(1-c) \log (1-c)
$$

that has been considered also for phase separation problems (Elliott \& Luckhaus, 1991). Indeed, in our analysis it is also possible to take $\chi$, instead of $I_{[0,1]}$, in (1.13). We notice anyway that this logarithmic potential is more usually considered in the non-double-well case (i.e. $j=\chi$ without the nonconvex term $j_{0}$ ). This means that the mixed configurations are assumed to attain a lower energy level than the pure ones, and this is not the case of our model.

Since the dependence on $\varphi$ in relation (1.12) for the free energy density $f$ is exactly the same as in the Warren-Boettinger model (Warren \& Boettinger, 1995), we proceed as in Kessler (2001) and Warren \& Boettinger (1995) to determine $f^{A}$ and $f^{B}$ and obtain

$$
-\frac{\partial f}{\partial \varphi}=F_{1}(\varphi)+c F_{2}(\varphi),
$$

where $F_{1}, F_{2}$ are smooth functions which vanish for $\varphi=0$ and $\varphi=1$. Hence, equation (1.9) for the phase field becomes

$$
\partial_{t} \varphi=M\left(\varepsilon_{\varphi}^{2} \Delta \varphi+F_{1}(\varphi)+c F_{2}(\varphi)\right) \quad \text { in } \Omega
$$

Moreover, with the choice (1.12) for $f$, equation (1.11) for the concentration can be read as

$$
\begin{aligned}
& \partial_{t} c-\operatorname{div}(\mu(\varphi, c) \nabla w)=0, \\
& w \in-\varepsilon_{c}^{2} \Delta c+g(\varphi)+\partial I_{[0,1]}(c)+j_{0}^{\prime}(c),
\end{aligned}
$$

where $g(\varphi)=f^{B}(\varphi)-f^{A}(\varphi)$ (note that $g^{\prime}(\varphi)=-F_{2}(\varphi)$ and $g(\varphi)=0$ for $\varphi=0$ and $\varphi=1)$ and the new auxiliary variable $w$ is often named as the chemical potential.

Finally, a thermodynamically reasonable choice for the mobility in the case where it only depends on the concentration is given by

$$
\mu=\mu(c)=c(1-c)
$$

(see Elliott \& Garcke, 1996, and references therein). This prescribes that the diffusion effect for $c$ vanishes in the pure components $A$ and $B$; however, we remark that (1.20) is generally assumed in case $j=\chi$ (cf. (1.15)), so that $\mu$ cancels out with the denominator of the term $\nabla j^{\prime}(c)$ and the degenerate character of the evolution of $c$ is actually lost. 
Hence, our mathematically easier choice of a nondegenerate $\mu$ seems not to be severely restrictive. In more detail, we assume $\mu$ to be a continuous, positive, and bounded function allowed to depend also on $\varphi$ as in Kessler et al. (1998) and Rappaz \& Scheid (2000). A realistic expression for $\mu$ is given by

$$
\mu(c, \varphi)=D(\varphi)\left(1-\alpha(2 c-1)^{2}\right), \quad \text { for } c \in[0,1],
$$

where $\alpha \in[0,1)$ is a given parameter, the case $\alpha=1$ giving the degeneration. The function $D$ is a non-decreasing positive and bounded function such that $D(0)=\mu_{s}>0$ and $D(1)=\mu_{l}>0$ are respectively the solid and liquid mobility coefficients with $\mu_{s} \leqslant \mu_{l}$.

Without any loss of generality, we put $M=\varepsilon_{c}^{2}=\varepsilon_{\varphi}^{2}=1$ so that, setting $\gamma:=j_{0}^{\prime}$ and $\beta:=\partial I_{[0,1]}$, the equations (1.17)-(1.19) reduce to (1.1)-(1.3), respectively. Let us also mention that for $\varphi=0$ and $\varphi=1$, we have $g(\varphi)=0$ and we recover the Cahn-Hilliard equation for $c$ with a concentration dependent mobility studied in Barrett \& Blowey (1999) (see also Elliott \& Garcke, 1996).

We finally quote the paper of Elder et al. (2001), where a coupled system with a nonconserved phase field and a conserved concentration field is considered. The authors deal with the same generic equations as (1.9), (1.11) but with a slightly different free energy density $f$ and they obtain sharp interface limits. In this concern, we analyse a singular limit problem related to the system (1.1)-(1.3), where the fourth-order diffusion term $-\Delta c$ and the derivative $\gamma(c)=j_{0}^{\prime}(c)$ of the nonconvex part of the mixing energy are set equal to 0 in the limit. In order to motivate this analysis, let us notice that the total free energy $\mathcal{F}$ of the system can be broken down into two contributions $\mathcal{F}_{\varphi}$ and $\mathcal{F}_{c}$, where

$$
\mathcal{F}_{\varphi}=\int_{\Omega}\left(\frac{\varepsilon_{\varphi}^{2}}{2}|\nabla \varphi|^{2}+(1-c) f^{A}(\varphi)+c f^{B}(\varphi)\right)
$$

is the excess energy due to solid-liquid phase mixing, indicating that the system wants to separate its liquid and solid phases $(\varphi=0$ and $\varphi=1)$, and

$$
\mathcal{F}_{c}=\int_{\Omega}\left(\frac{\varepsilon_{c}^{2}}{2}|\nabla c|^{2}+I_{[0,1]}(c)+j_{0}(c)\right)
$$

is the excess energy due to concentration phase mixing, indicating that the system wants to separate into pure element phases $(c=0$ and $c=1)$. The term $I_{[0,1]}(c)$ provides the physical barriers at $c=0,1$.

Up to now, we have considered that solid-liquid phase separation and concentration phase separation can be described by energies of comparable magnitude, or equivalently that they act at the same time scale. Nevertheless, it is reasonable to assume that in fact solid-liquid phase separation occurs much faster than the coarsening of concentration phases. One way of describing this situation is to consider that the full contribution of $\mathcal{F}_{c}$ to the energy is much smaller than the contribution of $\mathcal{F}_{\varphi}$, even infinitesimally smaller. This leads us to introduce the new energy

$$
\mathcal{F}_{\lambda}=\mathcal{F}_{\varphi}+\lambda \mathcal{F}_{c}
$$

and let the parameter $\lambda$ be very small and eventually converge to 0 . Actually, the introduction of this artificial parameter $\lambda$, which is convenient for the forthcoming analysis, 
is equivalent to rescaling both $\varepsilon_{c}^{2}$ and $\gamma$. Let us note that the constraint $I_{[0,1]}$ is not modified in the multiplication by $\lambda$ and hence it is conserved at the limit.

We are able to prove the convergence of the solutions of the original system (1.1)(1.3) to a weak solution of the limit one provided that $\mu$ is allowed to depend only on $\varphi$ (corresponding to $\alpha=0$ in (1.21)). Let us note that the limit system is formally equivalent to a Stefan problem with zero specific heat for the unknown $c$, which is coupled with the regular diffusion equation (1.1) for $\varphi$. Hence, at the limit and at least for $\beta=I_{[0,1]}$, the evolution of $c$ does no longer account for a separation of components, but just for a diffusive behaviour as $c$ stays in between the physical barriers $c=0,1$.

Outline of the paper. In the next section we provide some analytical preliminaries that are required for stating the precise mathematical abstract formulation of the problem. This is presented in Section 3 together with our main related results. In Section 4 we approximate the system by regularizing the subdifferential term, by use of the Yosida approximation. Then we exploit a Faedo-Galerkin technique and prove global existence by an a priori estimates and passage to the limit argument. Section 5 is devoted to the analysis of further properties of the solution, as additional regularity, continuous dependence on data, uniqueness, and physical interpretation. Finally, in Section 6, the singular limit problem is considered and a related convergence result is proved.

\section{Mathematical preliminaries}

Let $\Omega$ be a smooth, bounded, and connected domain in $\mathbb{R}^{d}, 1 \leqslant d \leqslant 3$, and let $T>0$. Set $\Gamma:=\partial \Omega, \Sigma:=\Gamma \times(0, T), Q_{t}:=\Omega \times(0, t)$ for $t \in(0, T]$, and $Q:=Q_{T}$. Set also $H:=L^{2}(\Omega)$ and $V:=H^{1}(\Omega)$ and endow the latter space with the usual scalar product

$$
((v, w)):=\int_{\Omega} v w \mathrm{~d} x+\int_{\Omega} \nabla v \cdot \nabla w \mathrm{~d} x
$$

We identify $H$ and its dual, in order that the compact inclusion $H \subset V^{\prime}$ holds and $\left(V, H, V^{\prime}\right)$ form a Hilbert triplet (Lions \& Magenes, 1972, p. 202). We denote by $(\cdot, \cdot)$ the scalar product of both $H$ and $H^{d}$ and by $|\cdot|$ the associated norms. Finally, we indicate by $\langle\cdot, \cdot\rangle$ the duality pairing between $V^{\prime}$ and $V$ and by $((\cdot, \cdot))_{*}$ the associated scalar product on $V^{\prime}$.

Let us introduce some notation for functions and functionals with zero mean value. Namely, for any $\zeta \in V^{\prime}$, let us set

$$
\begin{gathered}
\zeta_{\Omega}:=\frac{1}{|\Omega|}\langle\zeta, 1\rangle, \\
V_{0}^{\prime}:=\left\{\zeta \in V^{\prime}: \zeta_{\Omega}=0\right\}, \quad V_{0}:=V \cap V_{0}^{\prime} .
\end{gathered}
$$

Let now $0<\alpha \leqslant \mu_{0}$ be assigned constants and let

$$
\mu \in \operatorname{Lip}_{\text {loc }}\left(\mathbb{R}^{2}\right), \quad \text { with } \alpha \leqslant \mu \leqslant \mu_{0} \quad \text { a.e. in } \mathbb{R}^{2} \text {. }
$$


Let also $v, z: \Omega \rightarrow \mathbb{R}$ be measurable functions. Then, we can naturally associate with the couple $(v, z)$ the elliptic operator

$$
B_{(v, z)}: V \rightarrow V^{\prime}, \quad\left\langle B_{(v, z)} u, y\right\rangle:=\int_{\Omega} \mu(v, z) \nabla u \cdot \nabla y \mathrm{~d} x \quad \text { for } u, y \in V .
$$

Note indeed that

$$
\mu(v, z) \in L^{\infty}(\Omega) \quad \text { with } \alpha \leqslant \mu(v, z) \leqslant \mu_{0} \quad \text { a.e. in } \Omega .
$$

Analogously, we introduce the realization of the Laplace operator with homogeneous Neumann boundary conditions as

$$
B: V \rightarrow V^{\prime}, \quad\langle B u, y\rangle:=\int_{\Omega} \nabla u \cdot \nabla y \mathrm{~d} x \quad \text { for } u, y \in V .
$$

Clearly, $B, B_{(v, z)}$ map $V$ onto $V_{0}^{\prime}$ and their restrictions to $V_{0}$ turn out to be isomorphisms of $V_{0}$ onto $V_{0}^{\prime}$. Then, we can denote by $\mathcal{N}: V_{0}^{\prime} \rightarrow V_{0}$ the inverse of $B$ and by $\mathcal{N}_{(v, z)}$ : $V_{0}^{\prime} \rightarrow V_{0}$ the inverse of $B_{(v, z)}$. Just by applying the definition (2.5), one can readily check that for any $u \in V$ and $\zeta \in V_{0}^{\prime}$, there holds

$$
\left\langle B_{(v, z)} u, \mathcal{N}_{(v, z)} \zeta\right\rangle=\left\langle B_{(v, z)} \mathcal{N}_{(v, z)} \zeta, u\right\rangle=\langle\zeta, u\rangle .
$$

The next result (cf. Bonetti et al., 2003, Lemmas 2.2, 2.3) is an easy consequence of the Poincaré-Wirtinger inequality.

LEMMA 2.1 There exist constants $\kappa_{1}, \kappa_{2}>0$ depending only on $\Omega, \alpha, \mu$, such that for all $\zeta \in V_{0}^{\prime}$ and for all measurable functions $v, z: \Omega \rightarrow \mathbb{R}$ it is

$$
\left\|\mathcal{N}_{(v, z)} \zeta\right\|_{V} \leqslant \kappa_{1}\|\zeta\|_{V^{\prime}}, \quad\left\langle\zeta, \mathcal{N}_{(v, z)} \zeta\right\rangle \geqslant \kappa_{2}\|\zeta\|_{V^{\prime}}^{2}
$$

We also note that, as $v, z: Q \rightarrow \mathbb{R}$ are measurable functions, we can set, for a.e. $t \in(0, T)$, $B_{(v, z)}(t):=B_{(v(t), z(t))}$ and $\mathcal{N}_{(v, z)}(t):=\mathcal{N}_{(v(t), z(t))}$ and the operators $B_{(v, z)}$ and $\mathcal{N}_{(v, z)}$ can be actually intended to work on time-dependent functions. Namely, it is easy to show that (Bonetti et al., 2003, Lemma 3.1)

$$
\left\|B_{(v, z)}\right\|_{\mathcal{L}\left(L^{p}(0, T ; V), L^{p}\left(0, T ; V_{0}^{\prime}\right)\right)} \leqslant \mu_{0}, \quad\left\|\mathcal{N}_{(v, z)}\right\|_{\mathcal{L}\left(L^{p}\left(0, T ; V_{0}^{\prime}\right) L^{p}\left(0, T ; V_{0}\right)\right)} \leqslant \kappa_{1},
$$

where $\kappa_{1}$ is as in (2.9), $p \in[1, \infty]$, and by the notation $\mathcal{L}(X, Y)$ we mean the space of the linear and continuous operators between the Banach spaces $X$ and $Y$. Finally, let $J: H \rightarrow[0,+\infty]$ be a convex, lower semicontinuous, and proper function and $\mathcal{A}$ be its subdifferential, regarded as a (multivalued) operator of $H$ into itself. We need in the sequel a couple of integration by parts formulae, that are stated in the following two lemmas. We prove the first one, while the second is in Brezis \& Strauss (1973, Lemma 2).

Lemma 2.2 Let $T>0, u \in H^{1}\left(0, T ; V^{\prime}\right) \cap L^{2}(0, T ; V), \eta \in L^{2}(0, T ; V)$. Let also $\eta(t) \in \mathcal{A} u(t)$ for a.e. $t \in(0, T)$. Moreover, let us suppose that there exist $\kappa_{1}, \kappa_{2}>0$ such that

$$
J(v) \geqslant \kappa_{1}|v|^{2}-\kappa_{2} \quad \text { for all } v \in H .
$$


Then, the function $t \mapsto J(u(t))$ is absolutely continuous in $(0, T)$ and

$$
\int_{s}^{t}\left\langle\partial_{t} u(r), \eta(r)\right\rangle \mathrm{d} r=J(u(t))-J(u(s)) \quad \forall s, t \in(0, T) .
$$

Proof. Let us define a new convex functional $J_{\text {ext }}: V^{\prime} \rightarrow \mathbb{R}$ as

$$
J_{\text {ext }}(v):=J(v) \quad \text { if } v \in H, \quad J_{\text {ext }}(v):=+\infty \quad \text { otherwise. }
$$

Owing to (2.11), it is not difficult to prove that $J_{\text {ext }}$ is lower semicontinuous on $V^{\prime}$. Furthermore, by definition of subdifferential and using that $\eta \in V$, for any $\zeta \in H$ we have

$$
\langle\zeta-u, \eta\rangle=(\eta, \zeta-u) \leqslant J(\zeta)-J(u)=J_{\mathrm{ext}}(\zeta)-J_{\mathrm{ext}}(u) .
$$

Note that this relation still holds if $\zeta \in V^{\prime}$. Actually, due to (2.13), if $\zeta \in V^{\prime} \backslash H$, then $J_{\text {ext }}(\zeta)=+\infty$, and (2.14) is trivial. Then, denoting by $\mathcal{R}: V \rightarrow V^{\prime}$ the Riesz isomorphism, it is not difficult to infer that

$$
\mathcal{R} \eta \in \partial_{V^{\prime}} J_{\text {ext }}(u),
$$

where $\partial_{V^{\prime}} J_{\text {ext }}$ of course denotes the subdifferential of $J_{\text {ext }}$ with respect to the Hilbert structure of $V^{\prime}$. Then, Brezis (1973, Lemma 3.3, p. 73) can be applied in the space $V^{\prime}$. Furthermore, for all $s, t \in[0, T]$, a simple integration yields

$$
\begin{aligned}
\int_{s}^{t}\left\langle\partial_{t} u(r), \eta(r)\right\rangle \mathrm{d} r & =\int_{s}^{t}\left(\left(\partial_{t} u(r), \mathcal{R} \eta(r)\right)\right)_{*} \mathrm{~d} r \\
& =J_{\text {ext }}(u(t))-J_{\text {ext }}(u(s))=J(u(t))-J(u(s)),
\end{aligned}
$$

since it is known that $u(\cdot) \in H$ a.e. in $(0, T)$.

Lemma 2.3 Let $z, \xi \in H$, and let $J$ be the realization in $H$ of a convex, lower semicontinuous, and proper function $j: \mathbb{R} \rightarrow[0,+\infty]$, e.g.

$$
J(v):=\int_{\Omega} j(v(x)) \mathrm{d} x \quad \text { for } v \in H,
$$

where it is intended that the value of the integral might be $+\infty$ for some $v$. Then, let $\xi \in \partial J(z)$. Let also $\mu$ as in (2.4), $u, v, B_{(u, v)}$ as in (2.5). Finally, let $B_{(u, v)} z \in H$. Then, $\left(B_{(u, v)} z, \xi\right) \geqslant 0$.

\section{Main results}

Let us give the main assumptions on the data of the problem. Let $K>0$ and let

$$
\begin{aligned}
& F_{1}, F_{2}, \gamma, g \in W^{1, \infty}(\mathbb{R}), \\
& \left|F_{1}\right|,\left|F_{2}\right|,|\gamma|,|g|,\left|F_{1}^{\prime}\right|,\left|F_{2}^{\prime}\right|,\left|\gamma^{\prime}\right|,\left|g^{\prime}\right| \leqslant K \quad \text { a.e. in } \mathbb{R}, \\
& \varphi_{0} \in H, \quad c_{0} \in V, \\
& \beta \subset \mathbb{R} \times \mathbb{R} \quad \text { maximal monotone graph such that } 0 \in \beta(0) .
\end{aligned}
$$


Let us fix a convex and lower semicontinuous function $\psi: \mathbb{R} \rightarrow[0,+\infty]$ such that $\beta=\partial \psi$ and $\psi(0)=0$. Moreover, we recall that the domain of the graph $\beta$ is defined as

$$
D(\beta):=\{r \in \mathbb{R}: \beta(r) \neq \emptyset\} .
$$

Then, we also require

$$
\begin{gathered}
\psi\left(c_{0}\right) \in L^{1}(\Omega), \\
c_{\Omega} \in \operatorname{int} D(\beta), \quad \text { where } c_{\Omega}:=\left(c_{0}\right)_{\Omega} .
\end{gathered}
$$

We are now able to state our main existence result.

THEOREM 3.1 Let assumptions (2.4) and (3.1)-(3.6) hold. Then, there exists a quadruple $(\varphi, c, w, \xi)$ such that

$$
\begin{aligned}
& \varphi \in H^{1}\left(0, T ; V^{\prime}\right) \cap L^{2}(0, T ; V), \\
& c \in H^{1}\left(0, T ; V^{\prime}\right) \cap L^{\infty}(0, T ; V) \cap L^{2}\left(0, T ; H^{2}(\Omega)\right), \\
& w \in L^{2}(0, T ; V), \\
& \xi \in L^{2}(0, T ; H) .
\end{aligned}
$$

The quadruple $(\varphi, c, w, \xi)$ satisfies

$$
\begin{aligned}
\partial_{t} \varphi+B \varphi=F_{1}(\varphi)+c F_{2}(\varphi) & \text { in } V^{\prime} \text { a.e. in }(0, T), \\
\partial_{t} c+B_{(\varphi, c)} w=0 & \text { in } V^{\prime} \text { a.e. in }(0, T), \\
w=B c+\xi+\gamma(c)+g(\varphi) & \text { in } V^{\prime} \text { a.e. in }(0, T), \\
\xi \in \beta(c) & \text { a.e. in } Q, \\
\varphi(\cdot, 0)=\varphi_{0}(\cdot), \quad c(\cdot, 0)=c_{0}(\cdot) & \text { a.e. in } \Omega .
\end{aligned}
$$

Moreover, $c$ is a conserved order parameter, i.e.

$$
c(t)_{\Omega}=c_{\Omega} \quad \text { for all } t \in[0, T] .
$$

REMARK 3.2 Using (3.10) and the last of (3.8), one can see that (3.13) turns out to hold a.e. in $Q$. However, we prefer to state it in $V^{\prime}$, since this is the natural output space for the operator $B$.

Let us come to some regularity results.

THEOREM 3.3 In addition to (2.4) and (3.1)-(3.6), let

$$
\varphi_{0} \in V
$$

Then, the function $\varphi$ whose existence is ensured by Theorem 3.1 satisfies

$$
\varphi \in H^{1}(0, T ; H) \cap C^{0}([0, T] ; V) \cap L^{2}\left(0, T ; H^{2}(\Omega)\right) .
$$


THEOREM 3.4 In addition to (2.4) and (3.1)-(3.6), let

$$
\varphi_{0} \in H^{2}(\Omega), \quad \partial_{\mathbf{n}} \varphi_{0}=0 \quad \text { a.e. on } \Gamma
$$

where $\boldsymbol{n}$ denotes the outer unit normal vector to $\Omega$. Then, the function $\varphi$ given by Theorem 3.1 satisfies

$$
\varphi \in W^{1, \infty}(0, T ; H) \cap H^{1}(0, T ; V) \cap L^{\infty}\left(0, T ; H^{2}(\Omega)\right) \cap L^{2}\left(0, T ; H^{3}(\Omega)\right) .
$$

The following continuous dependence results hold in the regularity setting of Theorem 3.1, but only in case the function $\mu$ in (2.4) is replaced by a constant.

THEOREM 3.5 Let us assume (3.1)-(3.2) and (3.4), and two pairs of initial data $\left(\varphi_{0,1}, c_{0,1}\right)$ and $\left(\varphi_{0,2}, c_{0,2}\right)$, satisfying (3.3) and (3.5), (3.6). Moreover, let us assume that

$$
\left(c_{0,1}\right)_{\Omega}=\left(c_{0,2}\right)_{\Omega} .
$$

Then, let us consider the system given by (3.11), (3.13)-(3.15), and

$$
\partial_{t} c+B w=0 \quad \text { in } V^{\prime} \text { a.e. in }(0, T) \text {. }
$$

and, for $i=1,2$, let $\left(\varphi_{i}, c_{i}, w_{i}, \xi_{i}\right)$ be two solutions of such a system, related to the initial data $\left(\varphi_{0,1}, c_{0,1}\right)$ and $\left(\varphi_{0,2}, c_{0,2}\right)$, respectively. Moreover, let us assume that $R>0$ is a constant such that

$$
\left\|c_{1}\right\|_{L^{1}\left(0, T ; H^{2}(\Omega)\right)} \leqslant R
$$

Then, there exists a constant $C>0$ only depending on $\Omega, T, R$, and $K$, such that

$$
\begin{aligned}
& \left\|\varphi_{1}-\varphi_{2}\right\|_{C^{0}([0, T] ; H) \cap L^{2}(0, T ; V)}+\left\|c_{1}-c_{2}\right\|_{C^{0}\left([0, T] ; V^{\prime}\right) \cap L^{2}(0, T ; V)} \\
& \quad \leqslant C\left(\left|\varphi_{0,1}-\varphi_{0,2}\right|+\left\|c_{0,1}-c_{0,2}\right\|_{V^{\prime}}\right) .
\end{aligned}
$$

In particular, the solution to the modified system provided by Theorem 3.1 is unique.

Finally, let us prove that more restrictive assumptions on $F_{1}, F_{2}$ ensure that the component $\varphi$ of at least one solution admits a 'physical' interpretation as a phase variable.

THEOREM 3.6 Keeping the hypotheses of Theorem 3.1, let us also suppose

$$
\begin{gathered}
F_{1}(0)=F_{1}(1)=F_{2}(0)=F_{2}(1)=0, \\
0 \leqslant \varphi_{0} \leqslant 1 \quad \text { a.e. in } \Omega .
\end{gathered}
$$

Then, there exists at least a solution to (3.11)-(3.15) in the regularity setting of Theorem 3.1 whose component $\varphi$ fulfils

$$
\varphi \in L^{\infty}(Q), \quad \text { with } 0 \leqslant \varphi \leqslant 1 \quad \text { a.e. in } Q .
$$


REMARK 3.7 We observe that assumptions (3.4)-(3.6) actually generalize the natural physical assumptions on the graph $\beta$ and the initial datum $c_{0}$, that we now report in a rigorous mathematical form for the sake of completeness:

$$
\begin{aligned}
& 0 \leqslant c_{0} \leqslant 1 \quad \text { a.e. in } \Omega, \\
& 0<c_{\Omega}<1, \quad \text { where } c_{\Omega}:=\left(c_{0}\right)_{\Omega}, \\
& \beta=\partial I_{[0,1]} .
\end{aligned}
$$

Indeed, it is clear that, if (3.28)-(3.30) are fulfilled, then any solution $c$ to (3.13), (3.14) satisfies, in addition,

$$
c \in L^{\infty}(Q), \quad \text { with } 0 \leqslant c \leqslant 1 \quad \text { a.e. in } Q .
$$

\section{Proof of Theorem 3.1}

\subsection{Local existence}

We aim to prove existence for (3.11)-(3.15) by exploiting a Faedo-Galerkin approximation. Since the argument is standard we just sketch it briefly. First of all, we regularize the system by replacing $\beta$ with its Yosida approximation $\beta_{\varepsilon}$ (Brezis, 1973, p. 28). We denote by $\psi_{\varepsilon}$ the primitive of $\beta_{\varepsilon}$ such that $\psi_{\varepsilon}(0)=0$. Then, we take a complete and ordered system of eigenvectors $\left\{v_{i}\right\}$ of the standard Neumann problem for the Laplace operator. We set $V_{n}:=\operatorname{span}\left\{v_{1}, \ldots, v_{n}\right\}$ and $V_{\infty}:=\cup_{n=1}^{\infty} V_{n}$, which is a dense subspace of $V$, of course. For any $n \in \mathbb{N}$, we look for approximate solutions of the form

$$
\varphi_{\varepsilon}^{n}=\sum_{i=1}^{n} \varphi_{i}(t) v_{i}, \quad c_{\varepsilon}^{n}=\sum_{i=1}^{n} c_{i}(t) v_{i}, \quad w_{\varepsilon}^{n}=\sum_{i=1}^{n} w_{i}(t) v_{i} .
$$

Indeed, denoting by $\boldsymbol{\varphi}, \boldsymbol{c}, \boldsymbol{w}$ the vectors $\left\{\varphi_{i}\right\}_{i=1, \ldots, n},\left\{c_{i}\right\}_{i=1, \ldots, n},\left\{w_{i}\right\}_{i=1, \ldots, n}$, the finitedimensional approximation of (3.11)-(3.14) becomes

$$
\begin{aligned}
& \varphi^{\prime}=-\Lambda \varphi+\boldsymbol{F}(\boldsymbol{\varphi}, \boldsymbol{c}), \\
& \boldsymbol{c}^{\prime}=-M(\varphi, \boldsymbol{c}) \boldsymbol{w}, \\
& \boldsymbol{w}=\Lambda \boldsymbol{c}+\boldsymbol{\beta}_{\varepsilon}(\boldsymbol{c})+\gamma(\boldsymbol{c})+\boldsymbol{g}(\boldsymbol{\varphi}) .
\end{aligned}
$$

Here, the functions, $\boldsymbol{F}, \boldsymbol{\beta}_{\varepsilon}, \gamma$ and $\boldsymbol{g}$ are constructed in a natural way from $F_{1}$ and $F_{2}, \beta_{\varepsilon}$, $\gamma$ and $g$, respectively. Moreover, $\Lambda$ and $M$ are the elliptic matrices resulting from $B$ and $B_{(\varphi, c)}$.

It is easy to see that the right-hand sides of the system above are locally Lipschitz functions of their arguments. Then, approximating also the Cauchy condition (3.15) by choosing $\varphi_{0 n} \in V_{n}, c_{0 n} \in V_{n}$ with

$$
\varphi_{0 n} \rightarrow \varphi_{0} \quad \text { in } H, \quad c_{0 n} \rightarrow c_{0} \quad \text { in } V,
$$

and requiring of course

$$
\varphi_{\varepsilon}^{n}(0)=\varphi_{0 n}, \quad c_{\varepsilon}^{n}(0)=c_{0 n} \quad \text { a.e. in } \Omega,
$$

Cauchy's local existence theorem for ODEs yields a final time $T_{0}$, possibly $<T$, and a unique triplet $(\boldsymbol{\varphi}, \boldsymbol{c}, \boldsymbol{w}) \in C^{1}\left(\left[0, T_{0}\right] ; V_{n}^{3}\right)$, solving (4.2)-(4.4) up to $T_{0}$ and satisfying (4.6). 


\subsection{A priori estimates}

We now aim to prove some a priori estimates on the solution of the approximated system given by (4.2)-(4.4) and (4.6). Hence, let us rewrite (4.2)-(4.4) by replacing the expressions (4.1) therein:

$$
\begin{aligned}
\left\langle\partial_{t} \varphi_{\varepsilon}^{n}+B \varphi_{\varepsilon}^{n}, v\right\rangle=\left\langle F_{1}\left(\varphi_{\varepsilon}^{n}\right)+c_{\varepsilon}^{n} F_{2}\left(\varphi_{\varepsilon}^{n}\right), v\right\rangle & \forall v \in V_{n}, \quad \forall t \in(0, T), \\
\left\langle\partial_{t} c_{\varepsilon}^{n}+B_{\left(\varphi_{\varepsilon}^{n}, c_{\varepsilon}^{n}\right)} w_{\varepsilon}^{n}, v\right\rangle=0 & \forall v \in V_{n}, \quad \forall t \in(0, T), \\
\left\langle w_{\varepsilon}^{n}, v\right\rangle=\left\langle B c_{\varepsilon}^{n}+\beta_{\varepsilon}\left(c_{\varepsilon}^{n}\right)+\gamma\left(c_{\varepsilon}^{n}\right)+g\left(\varphi_{\varepsilon}^{n}\right), v\right\rangle & \forall v \in V_{n}, \quad \forall t \in(0, T) .
\end{aligned}
$$

In the sequel, $k$ will be a positive constant, possibly varying even inside a single row, but allowed to depend only on $\Omega, \alpha, \mu_{0}, K, T$ and, in particular, not on $T_{0}, n, \varepsilon$. Symbols like, for example, $k_{\sigma}$ are intended to mean that the constant $k_{\sigma}$ might also depend on one, or more, additional parameters (in this case $\sigma$ ). The elementary Young inequality

$$
r s \leqslant \sigma r^{2}+s^{2} / 4 \sigma \quad \text { for any } r, s \in \mathbb{R}, \sigma>0
$$

will be used repeatedly in the following. Finally, we observe that, since our estimates do not depend on $T_{0}$, the limit solution will turn out to be defined up to the final time $T$. For this reason, we shall directly work in the interval $(0, T)$.

Energy estimate. Take $t \in(0, T]$, choose $v=w_{\varepsilon}^{n}$ in (4.8) and $v=\partial_{t} c_{\varepsilon}^{n}$ in (4.9), integrate over $(0, t)$ and sum together the results. Owing to (2.4), observing that two terms cancel, and integrating in time the term with $\beta_{\varepsilon}$, it is easy to infer

$$
\begin{aligned}
\alpha \| & \nabla w_{\varepsilon}^{n} \|_{L^{2}\left(Q_{t}\right)}^{2}+\frac{1}{2}\left|\nabla c_{\varepsilon}^{n}(t)\right|^{2}+\int_{\Omega} \psi_{\varepsilon}\left(c_{\varepsilon}^{n}(t)\right) \mathrm{d} x \leqslant \frac{1}{2}\left|\nabla c_{0 n}\right|^{2} \\
& +\int_{\Omega} \psi_{\varepsilon}\left(c_{0 n}\right) \mathrm{d} x-\int_{0}^{t} \int_{\Omega} \gamma\left(c_{\varepsilon}^{n}\right) \partial_{t} c_{\varepsilon}^{n} \mathrm{~d} x \mathrm{~d} s-\int_{0}^{t} \int_{\Omega} g\left(\varphi_{\varepsilon}^{n}\right) \partial_{t} c_{\varepsilon}^{n} \mathrm{~d} x \mathrm{~d} s .
\end{aligned}
$$

The last two integrals on the right-hand side above can be estimated in several ways. For instance, splitting the former in the duality $\left(V, V^{\prime}\right)$, we have

$$
\left|\int_{0}^{t} \int_{\Omega} \gamma\left(c_{\varepsilon}^{n}\right) \partial_{t} c_{\varepsilon}^{n} \mathrm{~d} x \mathrm{~d} s\right| \leqslant \sigma\left\|\partial_{t} c_{\varepsilon}^{n}\right\|_{L^{2}\left(0, t ; V^{\prime}\right)}^{2}+k_{\sigma}+k_{\sigma}\left\|\nabla c_{\varepsilon}^{n}\right\|_{L^{2}\left(Q_{t}\right)}^{2}
$$

and, analogously,

$$
\left|\int_{0}^{t} \int_{\Omega} g\left(\varphi_{\varepsilon}^{n}\right) \partial_{t} c_{\varepsilon}^{n} \mathrm{~d} x \mathrm{~d} s\right| \leqslant \sigma\left\|\partial_{t} c_{\varepsilon}^{n}\right\|_{L^{2}\left(0, t ; V^{\prime}\right)}^{2}+k_{\sigma}+k_{\sigma}\left\|\nabla \varphi_{\varepsilon}^{n}\right\|_{L^{2}\left(Q_{t}\right)}^{2},
$$

where, of course, $\sigma$ is as in (4.10) and the above $k_{\sigma}$ also depend on the bound $K$ to $\gamma, g$ and their first derivatives.

Now, in order to estimate the norm of $\varphi_{\varepsilon}^{n}$ appearing in (4.13), we choose $v=\varphi_{\varepsilon}^{n}$ in (4.7) and integrate again over $(0, t)$. Taking advantage of (3.1), (3.2) again, it is immediate to infer

$$
\frac{1}{2}\left|\varphi_{\varepsilon}^{n}(t)\right|^{2}+\left\|\nabla \varphi_{\varepsilon}^{n}\right\|_{L^{2}\left(Q_{t}\right)}^{2} \leqslant \frac{1}{2}\left|\varphi_{0 n}\right|^{2}+k\left(1+\left\|\varphi_{\varepsilon}^{n}\right\|_{L^{2}\left(Q_{t}\right)}^{2}+\left\|c_{\varepsilon}^{n}\right\|_{L^{2}\left(Q_{t}\right)}^{2}\right) .
$$


We now have to estimate the norms of $\partial_{t} c_{\varepsilon}^{n}$ on the right-hand side of (4.12), (4.13). Then, note that $\lambda_{1}=0$, so that $1 \in V_{n}$ for every $n$ and we can choose $v=1$ in (4.8), obtaining

$$
\left\langle\partial_{t} c_{\varepsilon}^{n}, 1\right\rangle=0 .
$$

Thus, $\partial_{t} c_{\varepsilon}^{n} \in V_{n} \cap V_{0}$. Noting that $V_{n} \cap V_{0}=\operatorname{span}\left\{v_{2}, \ldots, v_{n}\right\}$ by orthogonality, one can readily see that the appropriate restriction of $\mathcal{N}$ is an isomorphism of $V_{n} \cap V_{0}$ onto itself (in coordinates it is the inverse of the diagonal matrix obtained by suppressing the first row and column of $\Lambda$ ). Hence, we can choose $v=\mathcal{N} \partial_{t} c_{\varepsilon}^{n}$ in (4.8). Integrating over $(0, t)$ and owing to (2.9), (2.10), (4.10), and to the Poincaré-Wirtinger inequality, we get

$$
\begin{aligned}
\left\|\partial_{t} c_{\varepsilon}^{n}\right\|_{L^{2}\left(0, t ; V^{\prime}\right)}^{2} & \leqslant k\left\|B_{\left(\varphi_{\varepsilon}^{n}, c_{\varepsilon}^{n}\right)} w_{\varepsilon}^{n}\right\|_{L^{2}\left(0, t ; V^{\prime}\right)}^{2} \leqslant k\left\|B_{\left(\varphi_{\varepsilon}^{n}, c_{\varepsilon}^{n}\right)}\left(w_{\varepsilon}^{n}-\left(w_{\varepsilon}^{n}\right)_{\Omega}\right)\right\|_{L^{2}\left(0, t ; V^{\prime}\right)}^{2} \\
& \leqslant k\left\|w_{\varepsilon}^{n}-\left(w_{\varepsilon}^{n}\right)_{\Omega}\right\|_{L^{2}(0, t ; V)}^{2} \leqslant k\left\|\nabla w_{\varepsilon}^{n}\right\|_{L^{2}\left(Q_{t}\right)}^{2} .
\end{aligned}
$$

Before collecting the above computations, we still have to recover the full $V$-norm of $c_{\varepsilon}^{n}$ on the left-hand side of (4.11) and we do this by noting that

$$
\begin{aligned}
\frac{1}{2}\left|c_{\varepsilon}^{n}(t)\right|^{2} & =\frac{1}{2}\left|c_{0 n}\right|^{2}+\int_{0}^{t}\left(\partial_{t} c_{\varepsilon}^{n}, c_{\varepsilon}^{n}\right) \mathrm{d} s \\
& \leqslant \frac{1}{2}\left|c_{0 n}\right|^{2}+\frac{1}{4}\left\|\partial_{t} c_{\varepsilon}^{n}\right\|_{L^{2}\left(0, t ; V^{\prime}\right)}^{2}+k\left\|c_{\varepsilon}^{n}\right\|_{L^{2}\left(Q_{t}\right)}^{2}+k\left\|\nabla c_{\varepsilon}^{n}\right\|_{L^{2}\left(Q_{t}\right)}^{2} .
\end{aligned}
$$

Now, let us multiply (4.11) by $m_{1}>0$ and (4.14) by $m_{2}>0$, where $m_{1}$ and $m_{2}$ will be chosen later. Then, we take the sum of the resulting relations and add also (4.15) and (4.16). Taking (4.12) and (4.13) into account, we infer

$$
\begin{aligned}
& m_{1} \alpha\left\|\nabla w_{\varepsilon}^{n}\right\|_{L^{2}\left(Q_{t}\right)}^{2}+\frac{m_{1}}{2}\left|\nabla c_{\varepsilon}^{n}(t)\right|^{2}+m_{1} \int_{\Omega} \psi_{\varepsilon}\left(c_{\varepsilon}^{n}(t)\right) \mathrm{d} x+\frac{m_{2}}{2}\left|\varphi_{\varepsilon}^{n}(t)\right|^{2} \\
& \quad+m_{2}\left\|\nabla \varphi_{\varepsilon}^{n}\right\|_{L^{2}\left(Q_{t}\right)}^{2}+\left\|\partial_{t} c_{\varepsilon}^{n}\right\|_{L^{2}\left(0, t ; V^{\prime}\right)}^{2}+\frac{1}{2}\left|c_{\varepsilon}^{n}(t)\right|^{2} \\
& \leqslant \\
& k_{\sigma, m_{1}, m_{2}}+\frac{m_{1}}{2}\left|\nabla c_{0 n}\right|^{2}+m_{1} \int_{\Omega} \psi_{\varepsilon}\left(c_{0 n}\right) \mathrm{d} x+k_{\sigma, m_{2}}\left\|c_{\varepsilon}^{n}\right\|_{L^{2}\left(Q_{t}\right)}^{2} \\
& \quad+k_{\sigma, m_{2}}\left\|\varphi_{\varepsilon}^{n}\right\|_{L^{2}\left(Q_{t}\right)}^{2}+\left(\frac{1}{4}+2 m_{1} \sigma\right)\left\|\partial_{t} c_{\varepsilon}^{n}\right\|_{L^{2}\left(0, t ; V^{\prime}\right)}^{2}+\left(m_{1} k_{\sigma}+k\right)\left\|\nabla c_{\varepsilon}^{n}\right\|_{L^{2}\left(Q_{t}\right)}^{2} \\
& \quad+m_{1} k_{\sigma}^{*}\left\|\nabla \varphi_{\varepsilon}^{n}\right\|_{L^{2}\left(Q_{t}\right)}^{2}+\frac{m_{2}}{2}\left|\varphi_{0 n}\right|^{2}+k^{*}\left\|\nabla w_{\varepsilon}^{n}\right\|_{L^{2}\left(Q_{t}\right)}^{2}+\frac{1}{2}\left|c_{0 n}\right|^{2},
\end{aligned}
$$

where $k_{\sigma}^{*}$ is the constant $k_{\sigma}$ multiplying the last norm in (4.13) and $k^{*}$ is the constant $k$ on the right-hand side of (4.15).

Now, let us choose successively $m_{1}, \sigma$, and $m_{2}$, in order that

$$
m_{1} \geqslant \frac{2 k^{*}}{\alpha}, \quad \sigma \leqslant \frac{1}{4 m_{1}}, \quad m_{2} \geqslant 2 m_{1} k_{\sigma}^{*} .
$$

Then, all the terms on the right-hand side of (4.17) are controlled. Indeed, (4.5) holds and we notice that, by (3.5) and Brezis (1973, Proposition 2.11, p. 39),

$$
\int_{\Omega} \psi_{\varepsilon}\left(c_{0 n}\right) \mathrm{d} x \leqslant 1+\int_{\Omega} \psi\left(c_{0}\right) \mathrm{d} x \leqslant k,
$$


at least for $n$ large enough, depending on $\varepsilon$. Hence, we readily see that Gronwall's lemma can be applied to the function

$$
t \mapsto\left\|c_{\varepsilon}^{n}(t)\right\|_{V}^{2}+\left|\varphi_{\varepsilon}^{n}(t)\right|^{2}
$$

in order to derive a bound. Since we need an estimate for the full $V$-norm of $w_{\varepsilon}^{n}$, we take $v=w_{\varepsilon}^{n}$ in (4.9) and integrate over $(0, t)$. Owing to the Lipschitz continuity of $\gamma, g$ and $\beta_{\varepsilon}$ and noting that, due to the estimates given by (4.17),

$$
\left|\int_{0}^{t}\left\langle B c_{\varepsilon}^{n}, w_{\varepsilon}^{n}\right\rangle \mathrm{d} s\right| \leqslant \frac{1}{2}\left\|\nabla w_{\varepsilon}^{n}\right\|_{L^{2}\left(Q_{t}\right)}^{2}+\frac{1}{2}\left\|\nabla c_{\varepsilon}^{n}\right\|_{L^{2}\left(Q_{t}\right)}^{2} \leqslant k,
$$

we readily get

$$
\left\|w_{\varepsilon}^{n}\right\|_{L^{2}(Q)}^{2} \leqslant k_{\varepsilon}+k
$$

where the constant $k_{\varepsilon}$ resulting from the term $\beta_{\varepsilon}\left(c_{\varepsilon}\right)$ is quadratically dependent on the Lipschitz constant of $\beta_{\varepsilon}$ and explodes as $\varepsilon \searrow 0$.

\subsection{Passage to the limit}

Passage to the limit as $n \nearrow \infty$. We now aim to pass to the limit firstly as $n \nearrow \infty$ and then as $\varepsilon \searrow 0$. Henceforth, all the convergence relations will be meant to hold up to the extraction of suitable subsequences, generally not relabelled. Then, from relations (4.17), (4.18), we see that there exists a triplet $\left(\varphi_{\varepsilon}, c_{\varepsilon}, w_{\varepsilon}\right)$, such that

$$
\begin{aligned}
\varphi_{\varepsilon}^{n} & \rightarrow \varphi_{\varepsilon} & & \text { weakly star in } L^{\infty}(0, T ; H) \cap L^{2}(0, T ; V), \\
c_{\varepsilon}^{n} & \rightarrow c_{\varepsilon} & & \text { weakly star in } H^{1}\left(0, T ; V^{\prime}\right) \cap L^{\infty}(0, T ; V), \\
w_{\varepsilon}^{n} & \rightarrow w_{\varepsilon} & & \text { weakly in } L^{2}(0, T ; V) .
\end{aligned}
$$

Then, standard interpolation and compact embedding results for vector-valued functions (see e.g. Simon, 1987, Section 8) ensure that

$$
c_{\varepsilon}^{n} \rightarrow c_{\varepsilon} \quad \text { strongly in } C^{0}([0, T] ; H)
$$

In order to derive some strong convergence for $\varphi_{\varepsilon}^{n}$ we need an estimate of its time derivative. Due to the finite-dimensional setting, we cannot proceed by a direct comparison in (4.7); then, we choose $v=\mathcal{N}\left(\partial_{t} \varphi_{\varepsilon}^{n}-\left(\partial_{t} \varphi_{\varepsilon}^{n}\right)_{\Omega}\right) \in V_{n}$ in (4.7) and integrate over $(0, t)$, $t \leqslant T$, deriving

$$
\begin{gathered}
\int_{0}^{t}\left\langle\partial_{t} \varphi_{\varepsilon}^{n}, \mathcal{N}\left(\partial_{t} \varphi_{\varepsilon}^{n}-\left(\partial_{t} \varphi_{\varepsilon}^{n}\right)_{\Omega}\right)\right\rangle \mathrm{d} s+\int_{0}^{t}\left\langle B \varphi_{\varepsilon}^{n}, \mathcal{N}\left(\partial_{t} \varphi_{\varepsilon}^{n}-\left(\partial_{t} \varphi_{\varepsilon}^{n}\right)_{\Omega}\right)\right\rangle \mathrm{d} s \\
=\int_{0}^{t}\left\langle F_{1}\left(\varphi_{\varepsilon}^{n}\right)+c_{\varepsilon}^{n} F_{2}\left(\varphi_{\varepsilon}^{n}\right), \mathcal{N}\left(\partial_{t} \varphi_{\varepsilon}^{n}-\left(\partial_{t} \varphi_{\varepsilon}^{n}\right)_{\Omega}\right)\right\rangle \mathrm{d} s .
\end{gathered}
$$


Note now that, since $\mathcal{N}\left(\partial_{t} \varphi_{\varepsilon}^{n}-\left(\partial_{t} \varphi_{\varepsilon}^{n}\right)_{\Omega}\right) \in V_{0}$ a.e. in $(0, T)$, we can rely on (2.9) and obtain

$$
\begin{aligned}
& \int_{0}^{t}\left\langle\partial_{t} \varphi_{\varepsilon}^{n}, \mathcal{N}\left(\partial_{t} \varphi_{\varepsilon}^{n}-\left(\partial_{t} \varphi_{\varepsilon}^{n}\right)_{\Omega}\right)\right\rangle \mathrm{d} s \\
& \quad=\int_{0}^{t}\left\langle\partial_{t} \varphi_{\varepsilon}^{n}-\left(\partial_{t} \varphi_{\varepsilon}^{n}\right)_{\Omega}, \mathcal{N}\left(\partial_{t} \varphi_{\varepsilon}^{n}-\left(\partial_{t} \varphi_{\varepsilon}^{n}\right)_{\Omega}\right)\right\rangle \mathrm{d} s \\
& \quad \geqslant k\left\|\partial_{t} \varphi_{\varepsilon}^{n}-\left(\partial_{t} \varphi_{\varepsilon}^{n}\right)_{\Omega}\right\|_{L^{2}\left(0, T ; V^{\prime}\right)}^{2} .
\end{aligned}
$$

Moreover, by (2.8), it is easy to check that

$$
\int_{0}^{t}\left\langle B \varphi_{\varepsilon}^{n}, \mathcal{N}\left(\partial_{t} \varphi_{\varepsilon}^{n}-\left(\partial_{t} \varphi_{\varepsilon}^{n}\right)_{\Omega}\right)\right\rangle \mathrm{d} s=\left|\varphi_{\varepsilon}^{n}(t)-\left(\varphi_{\varepsilon}^{n}\right)_{\Omega}\right|^{2}-\left|\varphi_{0 n}-\left(\varphi_{0 n}\right)_{\Omega}\right|^{2},
$$

and the latter norm is bounded, of course. Finally, the terms on the right-hand side of (4.23) can be split in the duality $\left(V^{\prime}, V\right)$ and estimated by taking account of (3.1)-(3.2), estimates (4.19), (4.20), and the continuous embedding $V \subset H$. This allows us to derive from (4.23), (4.24) the relation

$$
\left\|\partial_{t} \varphi_{\varepsilon}^{n}-\left(\partial_{t} \varphi_{\varepsilon}^{n}\right)_{\Omega}\right\|_{L^{2}\left(0, T ; V^{\prime}\right)}^{2} \leqslant k .
$$

Next, we notice that, $\left(\partial_{t} \varphi_{\varepsilon}^{n}\right)_{\Omega}$ is constant in $\Omega$ everywhere in $[0, T]$. Let us now take $v= \pm 1$ in (4.7) and note that, by (3.2) and the second of (4.20),

$$
\left|\left(\partial_{t} \varphi_{\varepsilon}^{n}\right)_{\Omega}(t)\right| \leqslant k\left(1+\left\|c_{\varepsilon}^{n}\right\|_{L^{\infty}\left(0, T ; L^{1}(\Omega)\right)}\right) \leqslant k
$$

for all $t \in[0, T]$. Then, (4.25) yields

$$
\partial_{t} \varphi_{\varepsilon}^{n} \rightarrow \partial_{t} \varphi_{\varepsilon} \quad \text { weakly in } L^{2}\left(0, T ; V^{\prime}\right)
$$

and, using (4.19) and Simon (1987, Section 8) again,

$$
\varphi_{\varepsilon}^{n} \rightarrow \varphi_{\varepsilon} \quad \text { strongly in } L^{2}(0, T ; H) \cap C^{0}\left([0, T] ; V^{\prime}\right) .
$$

Now, the boundedness and Lipschitz continuity of $F_{1}, F_{2}, \gamma, g$, and $\beta_{\varepsilon}$, together with relations (4.22) and (4.28), allow us to take the limits of the nonlinearities in (4.7) and (4.9). As for dealing with (4.8), we have to rewrite it as

$$
\left\langle\partial_{t} c_{\varepsilon}^{n}, v\right\rangle+\int_{\Omega} \mu\left(\varphi_{\varepsilon}^{n}, c_{\varepsilon}^{n}\right) \nabla w_{\varepsilon}^{n} \cdot \nabla v \mathrm{~d} x \quad \forall v \in V_{n} \quad \text { a.e. in }(0, T) .
$$

Then, we note that, by (2.4) and Lebesgue's dominated convergence theorem,

$$
\mu\left(\varphi_{\varepsilon}^{n}, c_{\varepsilon}^{n}\right) \rightarrow \mu\left(\varphi_{\varepsilon}, c_{\varepsilon}\right) \quad \text { weakly star in } L^{\infty}(Q), \quad \text { and strongly in } L^{p}(Q)
$$

for any $p<\infty$. Thus, recalling (4.21),

$$
\mu\left(\varphi_{\varepsilon}^{n}, c_{\varepsilon}^{n}\right) \nabla w_{\varepsilon}^{n} \rightarrow \mu\left(\varphi_{\varepsilon}, c_{\varepsilon}\right) \nabla w_{\varepsilon} \quad \text { weakly in } L^{2}(Q)
$$


and this permits us to pass to the limit in (4.8). We still have to prove the $H^{2}$ regularity for $c_{\varepsilon}$. Then, it is enough to set $v=B c_{\varepsilon}^{n}$ in (4.9), integrate in time, and note that, by monotonicity,

$$
\int_{0}^{t}\left\langle\beta_{\varepsilon}\left(c_{\varepsilon}^{n}\right), B c_{\varepsilon}^{n}\right\rangle \mathrm{d} s=\int_{0}^{t} \int_{\Omega} \beta_{\varepsilon}^{\prime}\left(c_{\varepsilon}^{n}\right)\left|\nabla c_{\varepsilon}^{n}\right|^{2} \mathrm{~d} x \mathrm{~d} s \geqslant 0
$$

and that

$$
\left|\int_{0}^{t}\left\langle w_{\varepsilon}^{n}, B c_{\varepsilon}^{n}\right\rangle \mathrm{d} s\right| \leqslant\left\|\nabla w_{\varepsilon}^{n}\right\|_{L^{2}\left(Q_{t}\right)}\left\|\nabla c_{\varepsilon}^{n}\right\|_{L^{2}\left(Q_{t}\right)} \leqslant k .
$$

We point out that this estimate depends just on the $L^{2}$-norm of $\nabla w_{\varepsilon}^{n}$ and not on the full $V$-norm of $w_{\varepsilon}^{n}$; in particular, the $k$ on the right-hand side does not explode as $\varepsilon \searrow 0$.

Finally, we observe that, if $v \in V_{\infty}$ is fixed, system (4.7)-(4.9) surely makes sense for sufficiently large $n$. Then, by the density of $V_{\infty}$ in $V$, in the limit we are allowed to take any $v \in V$ as a test function. As for the Cauchy conditions (4.6), they pass to the limit too, since (4.5), (4.22) and the second of (4.28) hold.

Passage to the limit as $\varepsilon \searrow 0$. We would like to repeat the above procedure; however, we can no longer take advantage of the Lipschitz continuity of $\beta_{\varepsilon}$ and in particular of the bound (4.18). Hence, we have to perform a new estimate of $\beta_{\varepsilon}\left(c_{\varepsilon}\right)$, consisting of a variant of an argument devised in Kenmochi et al. (1995, Lemma 5.2). Namely, we set $x_{\varepsilon}:=\left(\beta_{\varepsilon}\left(c_{\varepsilon}\right)\right)_{\Omega}$ and take $v=\beta_{\varepsilon}\left(c_{\varepsilon}\right)-x_{\varepsilon}$ in the $n$-limit of (4.9). Moreover, we choose $v=\mathcal{N}_{\left(\varphi_{\varepsilon}, c_{\varepsilon}\right)}\left(\beta_{\varepsilon}\left(c_{\varepsilon}\right)-x_{\varepsilon}\right)$ in (4.8), subtract from the above, and integrate over $(0, t)$, where $t \leqslant T$. Proceeding as in Colli et al. (2001, Section 4), we can prove that

$$
\left\|\beta_{\varepsilon}\left(c_{\varepsilon}\right)-x_{\varepsilon}\right\|_{L^{2}(Q)}^{2} \leqslant k .
$$

Note in particular that, by (2.10), (4.10), and the continuous embedding $V \subset H$,

$$
\int_{0}^{t}\left\langle\partial_{t} c_{\varepsilon}, \mathcal{N}_{\left(\varphi_{\varepsilon}, c_{\varepsilon}\right)}\left(\beta_{\varepsilon}\left(c_{\varepsilon}\right)-x_{\varepsilon}\right)\right\rangle \mathrm{d} s \leqslant \frac{1}{4}\left\|\beta_{\varepsilon}\left(c_{\varepsilon}\right)-x_{\varepsilon}\right\|_{L^{2}\left(Q_{t}\right)}^{2}+k\left\|\partial_{t} c_{\varepsilon}\right\|_{L^{2}\left(0, t ; V^{\prime}\right)}^{2}
$$

and the latter quantity is bounded by (4.20).

The second part of the procedure consists in the estimation of the average $x_{\varepsilon}$, that can be performed exactly as in Bonetti et al. (2003, Section 5.3). Observe that, at this step, hypothesis (3.6) turns out to be crucial. This means that, in the physical case $\beta=\partial I_{[0,1]}$, we cannot start by concentrations $c_{0}$ a.e. equal to 0 or to 1 . By this argument, it follows the existence of a function $\xi$ such that

$$
\beta_{\varepsilon}\left(c_{\varepsilon}\right) \rightarrow \xi \quad \text { weakly in } L^{2}(0, T ; H) .
$$

Now, we can improve the bound (4.18) by taking $v=w_{\varepsilon}$ in the $n$-limit of (4.9). We readily obtain

$$
\left\|w_{\varepsilon}\right\|_{L^{2}(Q)}^{2} \leqslant k
$$


where $k$ no longer depends on $\varepsilon$, thanks to (4.34). Now the passage to the limit as $\varepsilon \searrow 0$ can be performed as in the previous section and suitable limit functions $(\varphi, c, w)$ are obtained as limits of $\left(\varphi_{\varepsilon}, c_{\varepsilon}, w_{\varepsilon}\right)$, respectively. The only difference concerns the identification of the function $\xi$. Indeed, by (4.34), the analogue of (4.22), namely

$$
c_{\varepsilon} \rightarrow c \quad \text { strongly in } C^{0}([0, T] ; H),
$$

and the monotonicity argument of Barbu (1976, Proposition 1.1, p. 42), one can actually prove (3.14). Finally, to conclude the proof of Theorem 3.1, it suffices to show (3.16) and, of course, it is enough to take $v=1 \in V_{1}$ in (4.8) of the approximate statement, and pass to the limit in $\varepsilon, n$.

\section{Regularity and uniqueness}

Proof of Theorem 3.3. We derive additional a priori estimates, independent of $\varepsilon, n$, for the solutions of the system (4.7)-(4.9). We just give the highlights of this procedure, since it is rather similar to the analogous argument in Rappaz \& Scheid (2000, Section 3). Thus, we first have to take $v=\partial_{t} \varphi_{\varepsilon}^{n}$ on the right-hand side of (4.7) and integrate over $(0, t)$, for $t \leqslant T$. Then, performing standard integrations by parts, using the uniform boundedness of $F_{1}$ and $F_{2}$ and (4.22), and taking advantage of (3.17), it is easy to arrive at

$$
\left\|\varphi_{\varepsilon}^{n}\right\|_{H^{1}(0, T ; H)}+\left\|\varphi_{\varepsilon}^{n}\right\|_{L^{\infty}(0, T ; V)} \leqslant k .
$$

Of course, to make the procedure rigorous, we have to improve (4.5), by requiring

$$
\varphi_{0 n} \in V_{n}, \quad \varphi_{0 n} \rightarrow \varphi_{0} \quad \text { in } V .
$$

Finally, choosing $v=B \varphi_{\varepsilon}^{n}$ in (4.7) and working as above, we get the bound

$$
\left\|B \varphi_{\varepsilon}^{n}\right\|_{L^{2}(0, T ; H)} \leqslant k .
$$

The above relations can be taken into account in order to get the convergences yielding (3.18) at the limit. Indeed, the $H^{2}$-regularity follows from (5.3) and the standard elliptic regularity theorems, while the $C^{0}([0, T] ; V)$ regularity in (3.18) is a consequence of, for example, Baiocchi (1967, Lemma 6.3).

Proof of Theorem 3.4. Again, we proceed similarly to (Rappaz \& Scheid, 2000, Section 4). Anyway, our conditions on $c_{\varepsilon}^{n}$ are slightly different from those of Rappaz \& Scheid (2000); so, we briefly detail the computations. Thus, we take $v=B^{2} \varphi_{\varepsilon}^{n}$ in (4.7) and integrate again over $(0, t)$. Then, by the Gauss-Green formula we get

$$
\frac{1}{2}\left|B \varphi_{\varepsilon}^{n}(t)\right|^{2}+\left\|\nabla B \varphi_{\varepsilon}^{n}\right\|_{L^{2}\left(Q_{t}\right)}^{2} \leqslant \frac{1}{2}\left|B \varphi_{0 n}(t)\right|^{2}+\int_{0}^{t}\left\langle F_{1}\left(\varphi_{\varepsilon}^{n}\right)+c_{\varepsilon}^{n} F_{2}\left(\varphi_{\varepsilon}^{n}\right), B^{2} \varphi_{\varepsilon}^{n}\right\rangle \mathrm{d} s
$$

and we have to estimate the right-hand side above. The initial datum can be controlled just by postulating

$$
\varphi_{0 n} \in V_{n}, \quad \varphi_{0 n} \rightarrow \varphi_{0} \quad \text { in } H^{2}(\Omega) .
$$


Furthermore, integrating by parts the latter term in (5.4) and using (3.2) and (4.10), we easily get

$$
\begin{aligned}
& \int_{0}^{t}\left\langle F_{1}\left(\varphi_{\varepsilon}^{n}\right)+c_{\varepsilon}^{n} F_{2}\left(\varphi_{\varepsilon}^{n}\right), B^{2} \varphi_{\varepsilon}^{n}\right\rangle \mathrm{d} s \leqslant \frac{1}{2}\left\|\nabla B \varphi_{\varepsilon}^{n}\right\|_{L^{2}\left(Q_{t}\right)}^{2} \\
& \quad+k\left\|\nabla \varphi_{\varepsilon}^{n}\right\|_{L^{2}\left(Q_{t}\right)}^{2}+k\left\|\nabla c_{\varepsilon}^{n}\right\|_{L^{2}\left(Q_{t}\right)}^{2}+k \int_{0}^{t} \int_{\Omega}\left|c_{\varepsilon}^{n}\right|^{2}\left|\nabla \varphi_{\varepsilon}^{n}\right|^{2} \mathrm{~d} x \mathrm{~d} s .
\end{aligned}
$$

Of course, recalling (4.19), (4.20), we just have to control the last term. Thus, using some standard three-dimensional embedding theorems, we get

$$
\begin{aligned}
& k \int_{0}^{t} \int_{\Omega}\left|c_{\varepsilon}^{n}\right|^{2}\left|\nabla \varphi_{\varepsilon}^{n}\right|^{2} \mathrm{~d} x \mathrm{~d} s \leqslant k \int_{0}^{t}\left\|c_{\varepsilon}^{n}(s)\right\|_{L^{4}(\Omega)}^{2}\left\|\nabla \varphi_{\varepsilon}^{n}(s)\right\|_{L^{4}(\Omega)}^{2} \mathrm{~d} s \\
& \quad \leqslant k \int_{0}^{t}\left\|c_{\varepsilon}^{n}(s)\right\|_{H^{1}(\Omega)}^{2}\left\|\varphi_{\varepsilon}^{n}(s)\right\|_{H^{2}(\Omega)}^{2} \mathrm{~d} s \\
& \quad \leqslant k \int_{0}^{t}\left\|c_{\varepsilon}^{n}(s)\right\|_{H^{1}(\Omega)}^{2}\left|\varphi_{\varepsilon}^{n}(s)\right|^{2} \mathrm{~d} s+k \int_{0}^{t}\left\|c_{\varepsilon}^{n}(s)\right\|_{H^{1}(\Omega)}^{2}\left|B \varphi_{\varepsilon}^{n}(s)\right|^{2} \mathrm{~d} s .
\end{aligned}
$$

Now, the first integral on the right-hand side is bounded by (4.19)-(4.20), while we can control the latter with the first term on the left-hand side of (5.4) if we take advantage of the second of (4.20) and of the Gronwall lemma in the form of, for example, Brezis (1973, Lemma A.4, p. 156).

Then, passing to the limit we derive the third and the fourth of (3.20). Now, we have to choose $v=\partial_{t} B \varphi_{\varepsilon}^{n}$ in (4.7) and integrate again in time. Proceeding as before, we obtain

$$
\left\|\partial_{t} \nabla \varphi_{\varepsilon}^{n}\right\|_{L^{2}\left(Q_{t}\right)}^{2}+\frac{1}{2}\left|B \varphi_{\varepsilon}^{n}(t)\right|^{2} \leqslant \frac{1}{2}\left|B \varphi_{0 n}(t)\right|^{2}+\int_{0}^{t}\left\langle F_{1}\left(\varphi_{\varepsilon}^{n}\right)+c_{\varepsilon}^{n} F_{2}\left(\varphi_{\varepsilon}^{n}\right), \partial_{t} B \varphi_{\varepsilon}^{n}\right\rangle \mathrm{d} s
$$

and we readily see that the right-hand side can be estimated as above. In particular, working as in (5.6), (5.7), we arrive at

$$
\begin{aligned}
& \int_{0}^{t} \int_{\Omega} c_{\varepsilon}^{n} F_{2}^{\prime}\left(\varphi_{\varepsilon}^{n}\right) \nabla \varphi_{\varepsilon}^{n} \cdot \partial_{t} \nabla \varphi_{\varepsilon}^{n} \mathrm{~d} x \mathrm{~d} s \\
& \quad \leqslant \frac{1}{4}\left\|\partial_{t} \nabla \varphi_{\varepsilon}^{n}\right\|_{L^{2}\left(Q_{t}\right)}^{2}+k \int_{0}^{t}\left\|c_{\varepsilon}^{n}(s)\right\|_{H^{1}(\Omega)}^{2}\left(\left|\varphi_{\varepsilon}^{n}(s)\right|^{2}+\left|B \varphi_{\varepsilon}^{n}(s)\right|^{2}\right) \mathrm{d} s .
\end{aligned}
$$

Then, (5.8) yields the second regularity in (3.20). To prove the first one, we differentiate in time (4.7) and test the result by $\partial_{t} \varphi_{\varepsilon}^{n}$. Note that this procedure is rigorous. Indeed, referring to the formulation (4.2), we see that the right-hand side is at least locally $C^{0,1}$. Thus, we get

$$
\begin{gathered}
\frac{1}{2}\left|\partial_{t} \varphi_{\varepsilon}^{n}(t)\right|^{2}+\left\|\partial_{t} \nabla \varphi_{\varepsilon}^{n}\right\|_{L^{2}\left(Q_{t}\right)} \leqslant \frac{1}{2}\left|\partial_{t} \varphi_{\varepsilon}^{n}(0)\right|^{2}+\int_{0}^{t}\left\langle F_{1}^{\prime}\left(\varphi_{\varepsilon}^{n}\right) \partial_{t} \varphi_{\varepsilon}^{n}, \partial_{t} \varphi_{\varepsilon}^{n}\right\rangle \mathrm{d} s \\
+\int_{0}^{t}\left\langle c_{\varepsilon}^{n} F_{2}^{\prime}\left(\varphi_{\varepsilon}^{n}\right) \partial_{t} \varphi_{\varepsilon}^{n}, \partial_{t} \varphi_{\varepsilon}^{n}\right\rangle \mathrm{d} s+\int_{0}^{t}\left\langle\partial_{t} c_{\varepsilon}^{n} F_{2}\left(\varphi_{\varepsilon}^{n}\right), \partial_{t} \varphi_{\varepsilon}^{n}\right\rangle \mathrm{d} s
\end{gathered}
$$


and we have to bound the four terms on the right-hand side. As for the initial datum, we note that, by (4.7), (5.5), the second of (4.5), and the boundedness of $F_{1}$ and $F_{2}$,

$$
\left|\partial_{t} \varphi_{\varepsilon}^{n}(0)\right|^{2} \leqslant k\left(\left|B \varphi_{0 n}\right|^{2}+\left|c_{0 n}\right|^{2}+1\right) \leqslant k
$$

By Hölder's inequality and the continuous embedding $H^{2}(\Omega) \subset L^{\infty}(\Omega)$, we see that the first couple of integrals $I_{1}+I_{2}$ can be estimated as follows:

$$
\left|I_{1}+I_{2}\right| \leqslant k \int_{0}^{t}\left(1+\left\|c_{\varepsilon}^{n}(s)\right\|_{H^{2}(\Omega)}\right)\left|\partial_{t} \varphi_{\varepsilon}^{n}(s)\right|^{2} \mathrm{~d} s .
$$

The latter integral $I_{3}$ on the right-hand side of (5.10) has to be split in the duality between $V^{\prime}$ and $V$ and gives

$$
\begin{aligned}
\left|I_{3}\right| & \leqslant \frac{1}{2}\left\|\partial_{t} c_{\varepsilon}^{n}\right\|_{L^{2}\left(0, t ; V^{\prime}\right)}^{2}+\frac{1}{2}\left\|F_{2}\left(\varphi_{\varepsilon}^{n}\right) \partial_{t} \varphi_{\varepsilon}^{n}\right\|_{L^{2}(0, t ; V)}^{2} \\
& \leqslant k+k\left\|\partial_{t} \varphi_{\varepsilon}^{n}\right\|_{L^{2}(0, t ; V)}^{2}+k \int_{0}^{t} \int_{\Omega}\left|\nabla \varphi_{\varepsilon}^{n}\right|^{2}\left|\partial_{t} \varphi_{\varepsilon}^{n}\right|^{2} \mathrm{~d} x \mathrm{~d} s \\
& \leqslant k+k \int_{0}^{t}\left\|\varphi_{\varepsilon}^{n}\right\|_{H^{3}(\Omega)}^{2}\left|\partial_{t} \varphi_{\varepsilon}^{n}(s)\right|^{2} \mathrm{~d} x \mathrm{~d} s .
\end{aligned}
$$

Thus, collecting (5.10)-(5.14) and using the third of (3.8) and the fourth of (3.20), we see that Gronwall's lemma applies to $t \mapsto\left|\partial_{t} \varphi_{\varepsilon}^{n}(t)\right|^{2}$, so that the proof of Theorem 3.4 turns out to be complete.

We now come to the 'physical' interpretation of the solution and assume (3.25), (3.26) in addition to (3.1)-(3.6). Then, we modify $F_{1}$ and $F_{2}$, by effecting a truncation. Namely, we set, for $i=1,2$,

$$
\widetilde{F}_{i}(r):=0 \quad \text { if } r<0 \text { or } r>1, \quad \widetilde{F}_{i}(r):=F_{i}(r) \quad \text { if } 0 \leqslant r \leqslant 1 .
$$

Thanks to (3.25), it is clear that $\widetilde{F}_{1}, \widetilde{F}_{2}$ still satisfy (3.1), (3.2). Thus, Theorem 3.1 guarantees the existence of a solution to the system $(3.11)-(3.15)$, where $\widetilde{F}_{1}, \widetilde{F}_{2}$ replace $F_{1}, F_{2}$ in (3.11). Now, we state a maximum principle argument ensuring that, under the regularity assumptions of Theorem 3.1, any solution to the modified system (3.11)-(3.15) is actually a solution also to the original one.

LEMMA 5.1 Under the assumptions (3.1)-(3.6) and (3.25), (3.26), the component $\varphi$ of any solution to (3.11)-(3.15)-with $\widetilde{F}_{1}, \widetilde{F}_{2}$ in place of $F_{1}, F_{2}$ - satisfies

$$
0 \leqslant \varphi \leqslant 1 \quad \text { a.e. in } Q \text {. }
$$

We do not report the proof of this lemma, that is rather standard and actually identical to the proof of Rappaz \& Scheid (2000, Theorem. 3). Anyway, we note that Theorem 3.6 follows as an easy consequence. Moreover, we observe that of course assumption (3.2) on $g$ can be dropped in the statement of Theorem 3.6, that holds for any locally Lipschitz $g$.

REMARK 5.2 It is worthwhile discussing an important consequence of the above property. Of course, we would like to prove the well-posedness of the system (3.11)-(3.15) in the 
physical case ensuring the bounds $0 \leqslant \varphi, c \leqslant 1$ a.e. in $Q$. The above lemma guarantees that any solution of the truncated system is a solution of the original one in the very general regularity setting of Theorem 3.1. Of course, if we were able to show the uniqueness of the solution to the original system, this would mean that this unique solution is physically meaningful, since it has to coincide with a solution of the truncated system, which surely exists. However, the uniqueness result provided by Theorem 3.5 holds just in case $\mu$ is a constant function. Hence, we cannot exclude that there exists some solution to (3.11)(3.15) whose component $\varphi$ attains its values also outside [0, 1].

Proof of Theorem 3.5. Let us set $\varphi:=\varphi_{1}-\varphi_{2}, c:=c_{1}-c_{2}, w:=w_{1}-w_{2}, \xi:=\xi_{1}-\xi_{2}$, $\varphi_{0}:=\varphi_{0,1}-\varphi_{0,2}, c_{0}:=c_{0,1}-c_{0,2}$. Then, write (3.11) firstly for $\varphi_{1}, c_{1}$ and then for $\varphi_{2}, c_{2}$, take the difference, multiply it by $\varphi$, and integrate over $(0, t)$, for $t \leqslant T$. Then, owing to (3.1), (3.2), it is easy to get

$$
\begin{aligned}
& \frac{1}{2}|\varphi(t)|^{2}+\|\nabla \varphi\|_{L^{2}\left(Q_{t}\right)}^{2} \leqslant \frac{1}{2}\left|\varphi_{0}\right|^{2}+\frac{3 K}{2} \int_{0}^{t}|\varphi(s)|^{2} \mathrm{~d} s \\
&+\frac{K}{2} \int_{0}^{t}|c(s)|^{2} \mathrm{~d} s+\int_{0}^{t} \int_{\Omega}\left|c_{1}\right|\left|F_{2}\left(\varphi_{1}\right)-F_{2}\left(\varphi_{2}\right)\right||\varphi| \mathrm{d} x \mathrm{~d} s
\end{aligned}
$$

and by the continuous embedding $H^{2}(\Omega) \subset L^{\infty}(\Omega)$, holding for $d \leqslant 3$, the latter integral can be estimated as

$$
\leqslant K \int_{0}^{t}\left\|c_{1}(s)\right\|_{L^{\infty}(\Omega)}|\varphi(s)|^{2} \mathrm{~d} s \leqslant k \int_{0}^{t}\left\|c_{1}(s)\right\|_{H^{2}(\Omega)}|\varphi(s)|^{2} \mathrm{~d} s,
$$

where $K$ is as in (3.2). Now, take the difference of (3.13) written for the two solutions, multiply it by $c$, and integrate again over $(0, t)$. Then, note that, by (3.21) and (3.16), $c(s) \in V_{0}$ for a.e. $s \in[0, T]$ and it makes sense to test the difference of the relations (3.22) by $\mathcal{N} c$. Moreover, using Lemma 2.1 it is not difficult to prove that

$$
\int_{0}^{t}\left\langle c_{t}, \mathcal{N} c\right\rangle \geqslant \frac{C_{\alpha, \Omega, \mu}}{2}\|c(t)\|_{V^{\prime}}^{2}-\frac{C_{\alpha, \Omega}}{2}\left\|c_{0}\right\|_{V^{\prime}}^{2} .
$$

Thus, comparing with the relation obtained from (3.13) and exploiting the monotonicity of $\beta$, we readily obtain

$$
\frac{C_{\alpha, \Omega, \mu}}{2}\|c(t)\|_{V^{\prime}}^{2}+\|\nabla c\|_{L^{2}\left(Q_{t}\right)}^{2} \leqslant \frac{C_{\alpha, \Omega}}{2}\left\|c_{0}\right\|_{V^{\prime}}^{2}+k\|\varphi\|_{L^{2}\left(Q_{t}\right)}^{2}+k\|c\|_{L^{2}\left(Q_{t}\right)}^{2} .
$$

Then, we note that, by the compact embedding $V \subset H$ and the Poincaré-Wirtinger inequality, for any $\sigma>0$ there exists $k_{\sigma}>0$ such that

$$
\|c\|_{L^{2}\left(Q_{t}\right)}^{2} \leqslant \sigma\|\nabla c\|_{L^{2}\left(Q_{t}\right)}^{2}+k_{\sigma}\|c\|_{L^{2}\left(0, t ; V^{\prime}\right)}^{2} .
$$

Then, summing together the expressions (5.17) and (5.19), taking (5.18) and (5.20) into account, choosing $\sigma$ suitably small, and using hypothesis (3.23), we note that the Gronwall lemma in the form of, for example, Brezis (1973, Lemma A.4, p. 156) can be applied to the function

$$
t \mapsto|\varphi(t)|^{2}+\|c(t)\|_{V^{\prime}}^{2},
$$

so that relation (3.24) can be inferred by standard considerations. 
REMARK 5.3 In the physical case (3.30), or just assuming that $D(\beta)$ is bounded, assumption (3.23) can be avoided, since one can just take $R=\sup \{|r|, r \in D(\beta)\}$ to estimate the term in (5.18).

\section{Singular limit problem}

Let us now study the singular limit problem for (3.11)-(3.15) described in the Introduction. Henceforth, we assume that the function $\mu$ defined in (2.4) depends only on $\varphi$, corresponding to $\alpha=0$ in (1.21), take a parameter $\lambda>0$ that is supposed to go to 0 in the limit, and consider a solution $\left(\varphi_{\lambda}, c_{\lambda}, w_{\lambda}, \xi_{\lambda}\right)$ to the system

$$
\begin{aligned}
\partial_{t} \varphi_{\lambda}+B \varphi_{\lambda}=F_{1}\left(\varphi_{\lambda}\right)+c_{\lambda} F_{2}\left(\varphi_{\lambda}\right) & \text { in } V^{\prime} \text { a.e. in }(0, T), \\
\partial_{t} c_{\lambda}+B_{\varphi_{\lambda}} w_{\lambda}=0 & \text { in } V^{\prime} \text { a.e. in }(0, T), \\
w_{\lambda}=\lambda B c_{\lambda}+\xi_{\lambda}+\lambda \gamma\left(c_{\lambda}\right)+g\left(\varphi_{\lambda}\right) & \text { in } V^{\prime} \text { a.e. in }(0, T), \\
\xi_{\lambda} \in \beta\left(c_{\lambda}\right) & \text { a.e. in } Q, \\
\varphi_{\lambda}(\cdot, 0)=\varphi_{0}(\cdot), \quad c_{\lambda}(\cdot, 0)=c_{0}(\cdot) & \text { a.e. in } \Omega .
\end{aligned}
$$

Here, $\varphi_{0}, c_{0}, F_{1}, F_{2}, \gamma, g$ and $\beta$ are as in Theorem 3.1 that, of course, can be used to deduce the existence of such a solution. Moreover, the operator $B_{\varphi_{\lambda}}$ is defined as in (2.5) with the only difference that it has to depend only on one function $\varphi_{\lambda}$. Hence, also the inverse map $\mathcal{N}_{\varphi_{\lambda}}$ of $V_{0}^{\prime}$ to $V_{0}$ retains the natural properties stated in Section 2. We have the following result.

THEOREM 6.1 Beyond the above stated hypotheses, let us assume that there exist $k_{1}, k_{2}>$ 0 such that

$$
\psi(r) \geqslant k_{1} r^{2}-k_{2} \quad \forall r \in D(\psi) .
$$

Then, there exists a quadruple $(\varphi, c, w, \xi)$, such that, as $\lambda \searrow 0$, the following relations hold:

$$
\begin{aligned}
\varphi_{\lambda} \rightarrow \varphi & \text { weakly star in } H^{1}\left(0, T ; V^{\prime}\right) \cap L^{\infty}(0, T ; H) \cap L^{2}(0, T ; V), \\
c_{\lambda} \rightarrow c & \text { weakly star in } H^{1}\left(0, T ; V^{\prime}\right) \cap L^{\infty}(0, T ; H), \\
\lambda c_{\lambda} \rightarrow 0 & \text { strongly in } L^{2}(0, T ; V) \quad \text { and weakly in } L^{2}\left(0, T ; H^{2}(\Omega)\right), \\
w_{\lambda} \rightarrow w & \text { weakly in } L^{2}(0, T ; V), \\
\xi_{\lambda} \rightarrow \xi & \text { weakly in } L^{2}(0, T ; H) .
\end{aligned}
$$

Moreover, the quadruple $(\varphi, c, w, \xi)$ satisfies

$$
\begin{aligned}
\partial_{t} \varphi+B \varphi=F_{1}(\varphi)+c F_{2}(\varphi) & \text { in } V^{\prime} \text { a.e. in }(0, T), \\
\partial_{t} c+B_{\varphi} w=0 & \text { in } V^{\prime} \text { a.e. in }(0, T), \\
w=\xi+g(\varphi) & \text { a.e. in } Q, \\
\xi \in \beta(c) & \text { a.e. in } Q, \\
\varphi(\cdot, 0)=\varphi_{0}(\cdot), \quad c(\cdot, 0)=c_{0}(\cdot) & \text { a.e. in } \Omega .
\end{aligned}
$$


REMARK 6.2 We notice that (6.6) is a standard assumption, that ensures the wellposedness of this kind of degenerate parabolic systems (Damlamian, 1977; Kenmochi, 1990). Of course, it is verified in the physical case (3.30), where the second of (6.8) can be straightforwardly improved as

$$
c_{\lambda} \rightarrow c \quad \text { weakly star in } L^{\infty}(Q) \quad \text { and } \quad 0 \leqslant c \leqslant 1 \text { a.e. in } Q .
$$

Proof. As usual, we proceed by compactness methods and start by deriving one new a priori estimate. To this aim, test (6.1) by $\varphi_{\lambda}$ and integrate as usual over $Q_{t}, t \leqslant T$, easily obtaining

$$
\frac{1}{2}\left|\varphi_{\lambda}(t)\right|^{2}+\left\|\nabla \varphi_{\lambda}\right\|_{L^{2}\left(Q_{t}\right)}^{2} \leqslant \frac{1}{2}\left|\varphi_{0}\right|^{2}+k+k\left\|\varphi_{\lambda}\right\|_{L^{2}\left(Q_{t}\right)}^{2}+k\left\|c_{\lambda}\right\|_{L^{2}\left(Q_{t}\right)}^{2}
$$

Now, test (6.2) by $w_{\lambda}$ and (6.3) by $\partial_{t} c_{\lambda}$, integrate over $Q_{t}$ and sum the results. Note that this procedure makes sense as the right-hand side of (6.3) lies in $L^{2}(0, T ; V)$. Then, we formally have

$$
\begin{aligned}
& \alpha\left\|\nabla w_{\lambda}\right\|_{L^{2}\left(Q_{t}\right)}^{2}+\frac{\lambda}{2}\left|\nabla c_{\lambda}(t)\right|^{2}+\int_{\Omega} \psi\left(c_{\lambda}(t)\right) \mathrm{d} x \\
& \quad \leqslant \frac{\lambda}{2}\left|\nabla c_{0}\right|^{2}+\int_{\Omega} \psi\left(c_{0}\right) \mathrm{d} x+\lambda \int_{0}^{t}\left\langle\partial_{t} c_{\lambda}, \gamma\left(c_{\lambda}\right)\right\rangle \mathrm{d} s+\int_{0}^{t}\left\langle\partial_{t} c_{\lambda}, g\left(\varphi_{\lambda}\right)\right\rangle \mathrm{d} s
\end{aligned}
$$

Note anyway that the integration in time of the term with $\xi_{\lambda}$ has to be justified, and this can be done by applying Lemma 2.2 (in particular, the integration formula (2.12)) with the choices of

$$
\begin{gathered}
J(v):=\int_{\Omega}\left(\psi(v(x))+\frac{\lambda}{2}|\nabla v(x)|^{2}\right) \mathrm{d} x, \quad \text { for } v \in H, \\
u:=c_{\lambda}, \quad \eta:=w_{\lambda}-\lambda \gamma\left(c_{\lambda}\right)-g\left(\varphi_{\lambda}\right) .
\end{gathered}
$$

Indeed, it is easy to show that $\eta \in L^{2}(0, T ; V)$ for all $\lambda$ and that

$$
\eta(t) \in \partial J(u(t)) \quad \text { for a.e. } t \in(0, T)
$$

Finally, (2.11) is a consequence of (6.6), so that the lemma can be applied.

Now, proceeding as in (4.15), one readily sees that there exists a constant $k_{\alpha}>0$ such that

$$
\frac{\alpha}{2}\left\|\nabla w_{\lambda}\right\|_{L^{2}\left(Q_{t}\right)}^{2} \geqslant k_{\alpha}\left\|\partial_{t} c_{\lambda}\right\|_{L^{2}\left(0, t ; V^{\prime}\right)}^{2}
$$

Then, we have to split the last two integrals in (6.19) w.r.t. the duality $\left(V^{\prime}, V\right)$. Namely, for some $k^{*}$ dependent on $\alpha$, but not on $\lambda$, we have

$$
\lambda \int_{0}^{t}\left\langle\partial_{t} c_{\lambda}, \gamma\left(c_{\lambda}\right)\right\rangle \mathrm{d} s \leqslant \frac{k_{\alpha}}{4}\left\|\partial_{t} c_{\lambda}\right\|_{L^{2}\left(0, t ; V^{\prime}\right)}^{2}+k^{*} \lambda^{2}\left\|c_{\lambda}\right\|_{L^{2}(0, t ; V)}^{2}+k \lambda^{2}
$$


and

$$
\int_{0}^{t}\left\langle\partial_{t} c_{\lambda}, g\left(\varphi_{\lambda}\right)\right\rangle \mathrm{d} s \leqslant \frac{k_{\alpha}}{4}\left\|\partial_{t} c_{\lambda}\right\|_{L^{2}\left(0, t ; V^{\prime}\right)}^{2}+k^{*}\left\|\varphi_{\lambda}\right\|_{L^{2}(0, t ; V)}^{2}+k .
$$

Now, let us multiply (6.18) by $m>0$ (to be chosen at the end), and sum the result to (6.19), so that, collecting also (6.22)-(6.24), we infer

$$
\begin{aligned}
& \frac{m}{2}\left|\varphi_{\lambda}(t)\right|^{2}+\left(m-k^{*}\right)\left\|\nabla \varphi_{\lambda}\right\|_{L^{2}\left(Q_{t}\right)}^{2}+\frac{\alpha}{2}\left\|\nabla w_{\lambda}\right\|_{L^{2}\left(Q_{t}\right)}^{2} \\
& \quad+\frac{k_{\alpha}}{2}\left\|\partial_{t} c_{\lambda}\right\|_{L^{2}\left(0, t ; V^{\prime}\right)}^{2}+\frac{\lambda}{2}\left|\nabla c_{\lambda}(t)\right|^{2}+\int_{\Omega} \psi\left(c_{\lambda}(t)\right) \mathrm{d} x \\
& \leqslant \frac{m}{2}\left|\varphi_{0}\right|^{2}+k\left(1+m+\lambda^{2}\right)+\frac{\lambda}{2}\left|\nabla c_{0}\right|^{2}+\int_{\Omega} \psi\left(c_{0}\right) \mathrm{d} x+\left(m k+k^{*}\right)\left\|\varphi_{\lambda}\right\|_{L^{2}\left(Q_{t}\right)}^{2} \\
& \quad+\left(m k+k^{*} \lambda^{2}\right)\left\|c_{\lambda}\right\|_{L^{2}\left(Q_{t}\right)}^{2}+k^{*} \lambda^{2}\left\|\nabla c_{\lambda}\right\|_{L^{2}\left(Q_{t}\right)}^{2} .
\end{aligned}
$$

Now, using (6.6), we immediately have

$$
\int_{\Omega} \psi\left(c_{\lambda}(t)\right) \mathrm{d} x \geqslant k_{1}\left|c_{\lambda}(t)\right|^{2}-k_{2}
$$

so that, upon choosing $m \geqslant 2 k^{*}$, we see that, at least for $\lambda$ sufficiently small, Gronwall's lemma applies once more to

$$
t \mapsto\left|\varphi_{\lambda}(t)\right|^{2}+\left|c_{\lambda}(t)\right|^{2}+\lambda\left|\nabla c_{\lambda}(t)\right|^{2},
$$

so that (6.8), the second and the third of (6.7), and the first of (6.9) readily follow from (6.25). Moreover, the first of (6.7) can be deduced by a direct comparison in (6.1), while for the other relations it is necessary to repeat the argument leading to the estimation of $\xi_{\lambda}$ and this can be performed as in Section 4, with minor modifications. Note indeed that in this setting the function $\beta$ is no longer regular; hence, to integrate by parts the term with $B c_{\lambda}$, Lemma 2.3 has to be used. Actually, this procedure gives relation (6.11). Now, to deduce (6.10), it suffices to estimate the $L^{2}(0, T ; H)$ norm of $w_{\lambda}$. Thus, test $(6.3)$ by $w_{\lambda}$, integrate over $(0, t)$, and note that

$$
\left|\int_{0}^{t} \lambda\left\langle B c_{\lambda}, w_{\lambda}\right\rangle \mathrm{d} s\right| \leqslant k\left\|\nabla w_{\lambda}\right\|_{L^{2}\left(Q_{t}\right)}^{2}+\lambda^{2}\left\|\nabla c_{\lambda}\right\|_{L^{2}\left(Q_{t}\right)}^{2} \leqslant k,
$$

thanks to (6.25) and the first of (6.9). At this point, (6.10) is a consequence of the other convergence relations and the second of (6.9) can be proved by a comparison in (6.3).

Finally, we have to show that the limit functions $(\varphi, c, w, \xi)$ fulfil system (6.12)-(6.16) and actually this can be performed similarly as in the proof of Theorem 3.1, with the complication that (6.8) by (Simon, 1987, Corollary 4, Section 8) just implies

$$
c_{\lambda} \rightarrow c \quad \text { strongly in } C^{0}\left([0, T] ; V^{\prime}\right)
$$

so, we do not have pointwise convergence for $c_{\lambda}$. However, (6.7) yields

$$
\varphi_{\lambda} \rightarrow \varphi \quad \text { strongly in } L^{2}(0, T ; H) \text { and pointwise. }
$$


Thus, the continuity and boundedness of $F_{2}$, Lebesgue's dominated convergence theorem, and the second of (6.8) entail

$$
c_{\lambda} F_{2}\left(\varphi_{\lambda}\right) \rightarrow c F_{2}(\varphi) \quad \text { weakly in } L^{p}(Q) \text { for any } p \in[1,2)
$$

and this permits us to pass to the limit in (6.1). Moreover, the passage to the limit in (6.2), (6.3) does not present difficulties, since of course $\lambda \gamma\left(c_{\lambda}\right)$ tends to 0 , for example, strongly in $L^{\infty}(Q)$ and the Cauchy conditions (6.16) are recovered as in Section 4.

Thus, to conclude the proof, we just have to identify $\xi$, i.e. to show (6.15). Note that we cannot proceed as before, since we do not have the strong convergence of $c_{\lambda}$ in $L^{2}(Q)$. Hence, we have to test again (6.3) by $c_{\lambda}$ and integrate over $(0, T)$, deriving

$$
\begin{aligned}
\int_{0}^{T}\left(\xi_{\lambda}(t), c_{\lambda}(t)\right) \mathrm{d} t= & \int_{0}^{T}\left\langle c_{\lambda}(t), w_{\lambda}(t)\right\rangle \mathrm{d} t-\lambda \int_{0}^{T}\left|\nabla c_{\lambda}(t)\right|^{2} \mathrm{~d} t \\
& -\lambda \int_{0}^{T} \int_{\Omega} c_{\lambda} \gamma\left(c_{\lambda}\right) \mathrm{d} x \mathrm{~d} t-\int_{0}^{T} \int_{\Omega} c_{\lambda} g\left(\varphi_{\lambda}\right) \mathrm{d} x \mathrm{~d} t .
\end{aligned}
$$

Then, we take the limsup of the relation above as $\lambda \searrow 0$ and notice that, thanks to (6.28) and (6.10), it is

$$
\lim _{\lambda \searrow 0} \int_{0}^{T}\left\langle c_{\lambda}(t), w_{\lambda}(t)\right\rangle \mathrm{d} t=\int_{0}^{T}\langle c(t), w(t)\rangle \mathrm{d} t .
$$

Consequently, using the strong convergence in (6.29) and performing a comparison in the already deduced relation $(6.14)$, we derive

$$
\begin{aligned}
\underset{\lambda \searrow 0}{\limsup } \int_{0}^{T}\left(\xi_{\lambda}(t), c_{\lambda}(t)\right) \mathrm{d} t & \leqslant \int_{0}^{T}\langle c(t), w(t)\rangle \mathrm{d} t-\int_{0}^{T} \int_{\Omega} c g(\varphi) \mathrm{d} x \mathrm{~d} t \\
& =\int_{0}^{T}(\xi(t), c(t)) \mathrm{d} t,
\end{aligned}
$$

so that (6.15) is once more a consequence of Barbu (1976, Proposition 1.1, p. 42).

\section{Acknowledgement}

The work of DK is supported by the Swiss National Science Foundation.

\section{REFERENCES}

BAIOCCHI, C. (1967) Sulle equazioni differenziali astratte lineari del primo e del secondo ordine negli spazi di Hilbert. Ann. Mat. Pura Appl., 76, 233-304.

BARBU, V. (1976) Nonlinear Semigroups and Differential Equations in Banach Spaces. Leyden: Noordhoff.

BArRetT, J. W. \& Blowey, J. F. (1999) Finite element approximation of the CahnHilliard equation with concentration dependent mobility. Math. Comput., 68, 487-517.

Blowey, J. F. \& Elliott, C. M. (1991) The Cahn-Hilliard gradient theory for phase separation with non-smooth free energy. I. Mathematical analysis. Eur. J. Appl. Math., 2, 233-280.

Blowey, J. F. \& Elliott, C. M. (1992) The Cahn-Hilliard gradient theory for phase separation with non-smooth free energy. II. Numerical analysis. Eur. J. Appl. Math., 3, 147-179. 
Blowey, J. F. \& Elliott, C. M. (1993) Curvature dependent phase boundary motion and parabolic double obstacle problems. Degenerate Diffusions (Minneapolis, MN, 1991), 1960, IMA Vol. Math. Appl. Vol. 47. New York: Springer.

Blowey, J. F. \& Elliott, C. M. (1994) A phase field model with a double obstacle potential. Motion by Mean Curvature and Related Topics (Trento, 1992), 1-22. Berlin: de Gruyter.

Bonetti, E., Dreyer, W. \& Schimperna, G. (2003) Global solution to a generalized CahnHilliard equation with viscosity. Adv. Differ. Eqns, 8, 231-256.

BrezIS, H. (1973) Opérateurs Maximaux Monotones et Sémi-groupes de Contractions dans les Espaces de Hilbert (North-Holland Math. Studies, Vol. 5). Amsterdam: North-Holland.

Brezis, H. \& Strauss, W. A. (1973) Semi-linear second-order elliptic equations in $L^{1}$. J. Math. Soc. Japan, 25, 565-590.

CAHn, J. W. \& Hilliard, J. E. (1958) Free energy of a nonuniform system. I. Interfacial free energy. J. Chem. Phys., 28, 258-267.

Colli, P., Gilardi, G., Grasselli, M. \& Schimperna, G. (2001) The conserved phase field system with memory. Adv. Math. Sci. Appl., 11, 265-291.

Damlamian, A. (1977) Some results on the multi-phase Stefan problem. Commun. Partial Differ. Eqns, 2, 1017-1044.

Elder, K. R., Grant, M., Provatas, N. \& Kosterlitz, J. M. (2001) Sharp interface limits of phase field models. Phys. Rev. E, 64.

Elliott, C. M. \& GARCKe, H. (1996) On the Cahn-Hilliard equation with degenerate mobility. SIAM J. Math. Anal., 27, 404-423.

ELLIOTT, C. M. \& LUCKHAUS, S. (1991) A generalised diffusion equation for phase separation of a multi-component mixture with interfacial energy SFB756. University of Bonn. Preprint 195.

KENMOCHI, N. (1990) Neumann problems for a class of nonlinear degenerate parabolic equations. Differ. Integr. Eqns, 3, 253-273.

Kenmochi, N., NiezgódKa, M. \& PAwlow, I. (1995) Subdifferential operator approach to the Cahn-Hilliard equation with constraint. J. Differ. Eqns, 117, 320-356.

Kessler, D. (2001) Modelling, mathematical and numerical study of a solutal phase field model. Ph.D. Thesis, EPFL Lausanne, Switzerland.

KESSlER, D., KRÜGER, O. \& SCHEID, J.-F. (1998) Construction d'un modèle de champ de phase à température homogène pour la solidification d'un alliage binaire. (French), Internal Report 10/98 EPFL Lausanne. Switzerland.

Lions, J. L. \& MAgenes, E. (1972) Non-homogeneous Boundary Value Problems and Applications Vol. I. Berlin: Springer.

RAPPAZ, J. \& SCHEID, J.-F. (2000) Existence of solutions to a phase field model for the isothermal solidification process of a binary alloy. Math. Methods. Appl. Sci., 23, 491-513.

Simon, J. (1987) Compact sets in the space $L^{p}(0, T ; B)$. Ann. Mat. Pura Appl., 146, 65-96.

Warren, J. A. \& Boettinger, W. J. (1995) Prediction of dendritic growth and microsegregation patterns in a binary alloy using the phase-field model. Acta Metall. Mater., 43, 689-703.

Wheeler, A. A., Boettinger, W. J. \& McFadden, G. B. (1992) Phase-field model for isothermal phase transitions in binary alloys. Phys. Rev. A, 45, 7424-7439. 